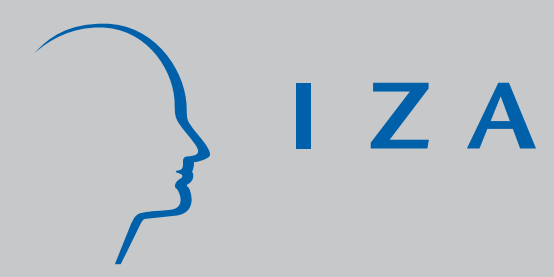

IZA DP No. 1260

Strategic Wage Setting and Coordination Frictions with Multiple Applications

Pieter A. Gautier

José L. Moraga-González

August 2004 


\title{
Strategic Wage Setting and Coordination Frictions with Multiple Applications
}

\author{
Pieter A. Gautier \\ Free University of Amsterdam, \\ Tinbergen Institute and IZA Bonn \\ José L. Moraga-González \\ Erasmus University of Rotterdam \\ and Tinbergen Institute
}

\section{Discussion Paper No. 1260 August 2004}

\author{
IZA \\ P.O. Box 7240 \\ 53072 Bonn \\ Germany \\ Phone: +49-228-3894-0 \\ Fax: +49-228-3894-180 \\ Email: iza@iza.org
}

\begin{abstract}
Any opinions expressed here are those of the author(s) and not those of the institute. Research disseminated by IZA may include views on policy, but the institute itself takes no institutional policy positions.

The Institute for the Study of Labor (IZA) in Bonn is a local and virtual international research center and a place of communication between science, politics and business. IZA is an independent nonprofit company supported by Deutsche Post World Net. The center is associated with the University of Bonn and offers a stimulating research environment through its research networks, research support, and visitors and doctoral programs. IZA engages in (i) original and internationally competitive research in all fields of labor economics, (ii) development of policy concepts, and (iii) dissemination of research results and concepts to the interested public.
\end{abstract}

IZA Discussion Papers often represent preliminary work and are circulated to encourage discussion. Citation of such a paper should account for its provisional character. A revised version may be available directly from the author. 


\section{ABSTRACT \\ Strategic Wage Setting and Coordination Frictions with Multiple Applications*}

We examine wage competition in a model where identical workers choose the number of jobs to apply for and identical firms simultaneously post a wage. The Nash equilibrium of this game exhibits the following properties: (i) an equilibrium where workers apply for just one job exhibits unemployment and absence of wage dispersion; (ii) an equilibrium where workers apply for two or for more (but not for all) jobs always exhibits wage dispersion and, typically, unemployment; (iii) the equilibrium wage distribution with a higher vacancy-to-unemployment ratio first-order stochastically dominates the wage distribution with a lower level of labor market tightness; (iv) the average wage is non-monotonic in the number of applications; (v) the equilibrium number of applications is non-monotonic in the vacancy-to-unemployment ratio; (vi) a minimum wage increase can be welfare improving because it compresses the wage distribution and reduces the congestion effects caused by the socially excessive number of applications; and (vii) the only way to obtain efficiency is to impose a mandatory wage that eliminates wage dispersion altogether.

JEL Classification: D4, J41, J64

Keywords: coordination frictions, internet, job search, minimum wage, wage dispersion

Corresponding author:

Pieter A. Gautier

Department of Economics

Free University of Amsterdam

De Boelelaan 1105

1081 HV Amsterdam

The Netherlands

Email: pgautier@feweb.vu.nl

\footnotetext{
* We have benefited from discussions with Jim Albrecht, Ken Burdett, Pierre Cahuc, Melvyn Coles, Andrea Galeotti, Rob van der Noll, Misja Nuyens, Vladimir Karamychev, Zsolt Sandor, Otto Swank, Susan Vroman and participants at the IZA workshop on search and matching models. Gautier acknowledges financial support from NWO through a VIDI grant.
} 


\section{Introduction}

At www.monster.com, unemployed workers can, at the cost of a few mouse clicks, observe all posted vacancies within a given region for a certain occupation. Most of the posted vacancies give a detailed job description but show no wage information. At the academic job market, candidates often send out more than 50 applications simultaneously without knowing precisely the wages they will be paid. The focus of this paper is on the features and efficiency properties of markets with the above characteristics. We present a model in which firms set wages, and workers apply to one or more jobs simultaneously. The number of applications a worker sends out when looking for a job and the wages that the firms set are endogenously determined in equilibrium. ${ }^{1}$ We are interested in the existence and the properties of Nash equilibria in this game.

In our model, the exogenous variables are: the relative size of the labor force, worker's application cost and the number of candidates that a firm can consider. Conditional on those variables we derive the number of matches, the wage distribution and the unemployment rate. The model has a number of novel features. First, the arrival rate of job offers is endogenous and sensitive to key labor market parameters such as the vacancy-to-unemployment ratio and the minimum wage. Secondly, we explicitly model the extent of congestion effects caused by the intensity of job search. This has important implications for the desirability of minimum wage increases or mandatory wages that eliminate wage dispersion altogether. Usually, search intensity is treated as a technology parameter (Pissarides, 2000), that increases the match probability for a worker. For the aggregate number of matches it does not matter whether the number of workers or the search intensity is doubled. In our model, a higher search intensity leads to more coordination problems between firms, as in Albrecht et al. (2004), and may thus lead to fewer matches; this may help explain the finding of Kuhn and Skuterud (2004) that Internet search does not reduce unemployment duration. Furthermore, since workers who receive multiple offers pick the job with the highest wage, wage dispersion increases the individual incentives to apply. In this respect our model differs from Shimer (2004). However, workers do not internalize the congestion effects of their applications and therefore, the equilibrium is not (constrained) efficient.

We study a market with $v \geq 1$ vacancies and $u \geq 2$ unemployed workers. We consider recruitment technologies $R_{m}$ where a firm offers the job to a first candidate picked at random (if it has

\footnotetext{
${ }^{1}$ The model is therefore not subject to the "partial-partial" critique of Rothschild (1973).
} 
any) and if it fails to hire her it offers the job to the next candidate (if present) and so on and so forth till its $m-t h$ candidate (if present). We focus on the two extreme cases of this recruitment technology. First, $R_{1}$, the one explored in Albrecht et al. (2003), where firms with candidates pick one worker at random, offer the job to her and the market closes. In the other extreme, $R_{u}$, where firms that fail to hire their first candidate can offer the job to their second candidate, and so on till the very last applicant they have. Workers who receive multiple offers take the one with the highest wage.

Depending on the cost involved in completing an application and on the minimum wage, there may be equilibria where workers either apply to just one job or apply to several jobs. When workers apply to just one job we get the standard Diamond (1971) result that the wage distribution is degenerate at the minimum wage. This follows from the fact that a firm only has an incentive to offer higher wages than other firms if this increases the hiring probability, but this cannot happen when all workers apply to just one job. We find that this type of result can only be sustained when, for a given level of the minimum wage, application costs are neither too high, which prevents worker participation, nor too low, which gives a worker incentives to deviate by applying to multiple jobs in order to increase her hiring probability. This contrasts with for example Acemoglu and Shimer (2000), where a monopsony equilibrium exists always.

When application cost are low, workers send multiple applications and the equilibrium is characterized by wage dispersion. The nature of the wage dispersion is similar to Burdett and Judd (1983), Lang (1991) and Acemoglu and Shimer (2000) where some workers observe more than one wage while others sample just one. In our model, some workers receive one offer and others receive two or more. In Burdett and Mortensen (1998), by contrast, wage dispersion arises because a firm's wage offer may arrive either at an employed worker (who are choosier) or at an unemployed worker, while in Albrecht and Axell (1984) it arises because workers have different reservation wages.

We now briefly discuss the unemployment features of the labor market equilibria. Recruitment technologies other than $R_{u}$ naturally lead to unemployment simply because workers cannot coordinate where they send their applications. But even under recruitment technology $R_{u}$, the most likely outcome is unemployment because the absence of it requires that workers apply to as many jobs as there are workers, which is unlikely to occur in large markets with costly applications.

Some of the mechanisms that are at work in our model are illustrated by a simple 3 worker-3 firm economy. In this context, we discuss the difference between the recruitment technologies $R_{1}$ and 
$R_{3}$. We find that the equilibrium characteristics are to a large extent invariant to the recruitment technology. The example also illustrates that individual incentives often lead to inefficient outcomes and that a minimum wage can be used to align the private and the social incentives. In the final part of the paper we examine large labor markets and we focus on $R_{1}$ because that allows us to solve the model for large $u$ and $v$. This gets extremely tedious under other recruitment mechanisms and the insights are similar as shown in the $3 \times 3$ market. In this context, we first explore the relationship between the equilibrium wage distribution and labor market tightness on the one hand, and the number of applications on the other hand. We find that, for a given number of applications, the equilibrium wage distribution with a higher vacancy-to-unemployment ratio first-order stochastically dominates the wage distribution with a lower labor market tightness. By contrast, a result in terms of first-order stochastic dominance cannot be established when comparing firm equilibria for different numbers of applications. We find that the average wage is non-monotonic (first increasing and then decreasing) in the number of applications. This is due to two facts that affect firm competitiveness in opposite directions: more applications increase the maximum number of offers a worker can receive but reduce the probability that a particular application leads to an offer.

Secondly, we explore how the equilibrium number of applications relates to labor market tightness. We find again a non-monotonic relationship. If the vacancy-to-unemployment ratio is initially small and it increases, then workers respond by sending more applications in equilibrium. By contrast, if the market tightness parameter is large to start with, an increase in the vacancyto-unemployment ratio leads to fewer applications. The intuition for this result comes from the incentives workers have to send out applications. On the one hand, more applications increase the chance to get at least one job offer. On the other hand, more applications increase the expected maximum wage offer. When the vacancy-to-unemployment ratio is very small, the wage distribution is skewed towards the minimum wage. As the $v / u$-ratio goes up, wages increase and workers marginal gains to apply rise. When the vacancy-to-unemployment rate is large, the wage distribution is compressed in the neighborhood of the competitive wage and then the marginal gains to apply fall as the vacancy-to-unemployment rate increases further.

We then study the relationship between the equilibrium number of applications and the minimum wage. Again, the way the minimum wage affects workers incentives to send out applications hinges upon labor market tightness: when there are few (many) firms per worker, an increase in the 
minimum wage leads to more (less) applications. The intuition for this result is related to the fact that the wage distribution and the minimum wage keep a monotonic relationship so, for example, when the wage distribution is already quite skewed towards the competitive wage, an increase in the minimum wage reduces workers incentives to apply. This motivates the question whether the minimum wage can be a useful policy instrument to enhance efficiency in our labor market.

To pursue this line of inquiry, we turn to examine whether private incentives lead to aggregate efficiency. We first observe that, irrespective of the level of the minimum wage, the number of applications workers send in equilibrium is excessive from a social welfare perspective. The idea here is that an individual worker does not internalize the effects that his/her applications cause on the other workers. This result contrasts with Acemoglu and Shimer (2000), where there is too little search in equilibrium because some workers free-ride on the search of others. The difference in results arises due to the additional motives workers have to send out applications in our setting: higher search intensity increases not only the expected wage but also the hiring probability. It then follows that an increase in the minimum wage is desirable if the vacancy-to-unemployment ratio is relatively large because in that case the equilibrium number of applications is reduced and welfare rises. This mechanism is new to our knowledge. When there is already a lot of competition for workers, extra applications are not desirable. Introducing a minimum wage reduces the number of applications by compressing the wage distribution and this can increase employment by reducing coordination frictions. By contrast, if the number of vacancies is small relative to the number of workers, then a minimum wage increase gives workers incentives to send more applications in equilibrium, which worsens the situation. We show that (constrained) efficiency can be achieved in our setting by imposing a particular mandatory wage that eliminates wage dispersion altogether and consequently one of the motives workers have to send applications.

The last issue we examine is how our equilibrium is affected by free entry of vacancies. We first explore the relationship between firm's incentives to enter the economy and the minimum wage for a given number of applications. Not surprisingly, we find that entry incentives are monotonically decreasing in the minimum wage. Secondly, we explore how firm entry is affected by the number of applications workers send out. We find that firm's incentives to enter are non-monotonic (first decreasing then increasing) in the number of applications. Thirdly, we find that, from the point of view of social welfare, entry is excessive if the minimum wage is low, while it is insufficient if the minimum wage is large. Finally, we look at how the minimum wage affects the free-entry 
equilibrium with an endogenous number of applications. Since the equilibrium conditions for labor market tightness and the number of applications are highly non-linear, the long-run implication of changes in the minimum wage are highly sensitive to the market's initial conditions. In the long-run equilibrium, an increase in the minimum wage always leads to exit and, if initially there are sufficiently many firms in the market, to more applications, otherwise to fewer applications.

Our analysis is further related to the consumer search models of Burdett and Judd (1983) and Janssen and Moraga-González (2004). The fundamental difference between those models and our labor market model is that here vacancies can only be occupied by a single worker whereas in the goods market, rationing is usually less important. Workers anticipate the labor market frictions that arise from rationing and send an excessive amount of applications, which in turn may amplify the extent of the frictions.

The nature of unemployment in our model is similar to the directed search models of Montgomery (1991), Burdett, Shi, Wright (2001) and Albrecht et al. (2003). When workers apply to one job only, some vacancies receive multiple applications while others receive none. Accordingly, some workers receive no offers and remain unemployed while others receive multiple offers. Albrecht et al. (2003) show that when workers apply to multiple jobs, this coordination failure does not disappear but becomes less important. Our work is important in this sense because workers do not only take into account the magnitude of these frictions but also the wage they expect to get when they decide upon the number of applications to send out. Another difference is that in our model, search is random, capturing the idea that there are characteristics about the job that the worker only finds out after the firm has been contacted. ${ }^{2}$ This generates atomless wage distributions in equilibrium while they derive two-point distributions. Whether search is random or directed is ultimately an empirical question. However casual evidence shows that some job advertisements do post a wage and, as in the example above, some don't.

The paper is organized as follows. Section 2 discusses the basic game. In section 3 we provide some properties of the equilibria. In section 4 we fully solve a $3 \times 3$ example for both $R_{1}$ and $R_{3}$. This simple example captures the main mechanisms that are at work and shows that different recruiting technologies lead to similar insights. Section 5 discusses a number of general results for large labor markets and some policy implications. Finally, section 6 concludes.

\footnotetext{
${ }^{2}$ For example, even though assistant professors may have some idea about the starting salaries at different universities, the amount of effort they have to put in the job depends on the teaching load, types of courses that must be taught, management duties, etc., which typically are revealed after the contact has taken place.
} 


\section{The labor market}

We consider a single period labor market model with $u \geq 2$ risk neutral unemployed workers and $v \geq 1$ risk neutral vacancies. Firms, indexed by the subscript $i$, post wages. We assume that each firm has exactly one vacancy. A strategy for firm $i$ is a wage offer, denoted $F_{i}(w)$. Wages must be posted above the minimum wage, denoted $\underline{w}$; this enables us to study the effects of minimum wage policies. ${ }^{3}$ When a worker is matched with a vacancy they produce one unit of output.

Workers, indexed by the subscript $j$, who manage to get a job receive a gross utility equal to the wage $w$ they are paid. To be able to receive wage offers, workers must send applications to the vacancies and complete the recruitment procedures. A typical worker knows the location of firms but she only learns about the wage of a firm after she has applied there and her application results in a wage offer. Applying to a job is costly. We assume that it costs a worker $k>0$ to complete and submit an application. Let $a$ denote the number of applications that a worker sends to different vacancies. A strategy for a worker $j$ is the number of applications to send out: $a_{j}=\{1,2, \ldots, v\}$. If a worker receives two or more wage offers, she chooses the vacancy that offers the highest wage.

Workers and firms play a simultaneous move game. A firm chooses a wage offer taking as given the wage offers of the rest of the firms as well as the number of applications sent by the workers. A worker decides how many applications to send taking as given the wages offered by the firms as well as the number of applications sent by the other workers. Once applications and wages have been decided upon, a firm obtaining multiple applications randomly picks a candidate and offers the job to her. If the candidate has no better offers, she accepts it while if she has better offer(s) she rejects it. Whether the firm can in the latter case go to its next candidate(s) depends on the degree of market frictions. To capture this idea we assume the following mechanism for the recruitment of workers:

Definition 1 Let $R_{m}$ be a recruitment technology. Under $R_{m}$, a firm that fails to hire its first

\footnotetext{
${ }^{3}$ Alternatively, nothing is lost when $\underline{w}$ is interpreted as unemployment income. Then, wages must be quoted above $\underline{w}$ to attract workers, and changes in $\underline{w}$ enable us to examine the implications of unemployment benefits.

${ }^{4}$ We are limiting the workers' strategy set in the sense that a worker cannot address her applications to particular vacancies. The implication of this assumption is that if a worker sends $a$ applications, the probability that a randomly selected vacancy gets an application from the worker in question is $a / v$. Since we focus on symmetric equilibria where identical workers use the same strategies, this is not restrictive. Equilibria where, say, worker 1 applies with probability one to firm $A$ and worker 2 with probability 1 to firm $B$ etc. are not so interesting because they require a lot of coordination, which is difficult to achieve, in particular when the labor market is large. Furthermore, we assume that workers play pure strategies in terms of the number of applications they send out.
} 
candidate (if any) can make an offer to its second candidate (if any), and so on till its $m$ - th candidate (if any), $m=1,2,3, \ldots, u$.

In Albrecht et al. $(2003,2004)$ it is assumed that screening a worker takes one period. This recruitment technology, labelled $R_{1}$, captures a situation where frictions are quite large so that a firm that fails to hire its first candidate closes its vacancy. The other extreme case is a situation where screening is costless so that a firm that fails to hire its first candidate can always go back to the next candidate(s). We label this screening technology, $R_{u}$ because the maximum number of candidates a firm may have is $u$. The hiring procedure under $R_{u}$ stops when all firms with applicants have hired a worker that did not get hired by some other firm. Obviously, in markets with relatively high frictions $R_{1}$ makes more sense that $R_{u}$, while the opposite holds true in markets with no frictions. Even though in general we expect intermediate technologies $R_{2}, \ldots, R_{u-1}$ to be in place in real world markets, we focus on the two extreme cases $R_{1}$ and $R_{u}$ in the remainder of the paper. One of the results we obtain is that these different recruitment technologies lead to similar qualitative results, so one would expect the same economic trade-offs to appear for the other screening technologies.

We focus on symmetric Nash equilibria of the game we described above. Asymmetric equilibria will in general exist but are not so interesting in large labor markets because they require a lot of coordination of the agents.

\section{$3 \quad$ Equilibria}

Our first result characterizes the number of applications $a$ that can be part of an equilibrium for different recruitment technologies.

Lemma 1 Under $R_{u}$, only $a \leq \min \{u, v\}$ can be part of an equilibrium, while under $R_{m}$ all $a \leq v$ can be part of an equilibrium, $m=1,2,3, \ldots, u-1$.

Proof. Consider $R_{u}$. Assume first that $\min \{u, v\}=v$. Since workers do not send multiple applications to the same firm it trivially follows that $a \leq v$. Assume now that $\min \{u, v\}=u$. We now prove that $a>u$ cannot be part of an equilibrium. Suppose, on the contrary that $a \geq u+1$. In that case all the workers as a collective would be in contact with a number of vacancies equal to or greater than $u+1$. Then, it is readily seen that firms' wage setting must be competitive, i.e., $w_{i}=1, i=1,2, \ldots, v$. But then any worker would gain by reducing the number of applications to $u$. 
Under $R_{m}, m=1,2,3, \ldots, u-1$, this argument fails because in that case, even if all the workers are in contact with a number of vacancies equal to or greater than $u+1$, the probability a given worker gets a job is strictly less than 1 . As a result, any number of applications $a \leq v$ can potentially be part of an equilibrium.

Our next result states some general features of wage equilibria.

Proposition 1 For any $u, v$ and $R_{m}$, (i) if an equilibrium exists where workers send just one application, the equilibrium wage distribution must be degenerate at the minimum wage $\underline{w}$; (ii) if an equilibrium exists where $v>a \geq 2$ then there must be wage dispersion and the wage distribution must be atomless; (iii) for any $u$ and $v$, if an equilibrium exists where $a=v=\min \{u, v\}$, the equilibrium wage distribution must be degenerate at the minimum wage $\underline{w}$ under recruitment technology $R_{u}$ and atomless under $R_{1}, R_{2}, \ldots, R_{u-1}$.

The proof, which is in the appendix, goes along the following lines. (i) When $a=1$, the probability that a given worker also gets an offer from a competing firm is 0 , hence no firm has an incentive to offer $w>\underline{w}$. So when $a=1$ the equilibrium wage distribution must be degenerate at $w=\underline{w}$. (ii) When $\min \{u, v\}>a \geq 2$, we can prove the statement above by contradiction. The proof is similar to Burdett and Judd (1983) and Lang (1991). Assume that all firms post some wage $w$ in $[\underline{w}, 1)$ in equilibrium. First, we show that there is always a positive probability that a vacancy remains unmatched. Second, we establish the contradiction. Equilibrium profits are: $\pi_{i}=\rho(1-w)$, where $\rho$ is the probability to fill a vacancy. We show that a firm, say $i$, can do better by deviating and offer a higher wage, say $w+\varepsilon$. The firm in question will then always succeed in hiring a worker, provided that it obtains at least an application. This occurs with a probability greater than $\rho$. By deviating, the firm therefore makes profits which are strictly higher than $\pi_{i}$ for sufficiently small $\varepsilon>0$. This argument rules out all wages in $[\underline{w}, 1)$. It remains to be proved that a wage distribution degenerated at $w=1$ cannot be an equilibrium either when $\min \{u, v\}>a \geq 2$. Note that in this case firms would make zero profits in equilibrium. If a firm deviates and offers a lower wage, say $\underline{w}$, this firm would obtain a profit $\pi_{i}^{D}=\rho^{D}(1-\underline{w})$, where $\rho^{D}$ is the probability that the deviant is matched with a worker. To rule out this case we show that $\rho^{D}>0$. We finally rule out mass points with similar arguments. (iii) Under $R_{U}$, if $a=v \leq u$, each firm gets $u$ applications and hires a worker with probability 1 and no firm has an incentive to offer a wage above the minimum 
wage. Under $R_{1}, \ldots, R_{u-1}$, there is always competition for workers and therefore the arguments in (ii) also hold here.

Proposition 1 highlights the influence of multiple applications by workers on wage setting and vice versa. Workers are more likely to send multiple applications in equilibrium when there is wage dispersion. Moreover, wage setting can only be dispersed if a firm faces a positive probability that an applicant is hired by some other firm, which necessarily requires that the applicant sends multiple applications. Therefore, wage dispersion and multiple applications are phenomena that are typically observed together. It is worth to remark that an equilibrium where wages are competitive does not exist in this market.

Our next result discusses general unemployment features of equilibria. If the number of vacancies is smaller than the number of unemployed workers then unemployment arises trivially. More interestingly:

Proposition 2 Let $v \geq u$. If an equilibrium exists where $a<u$, then unemployment arises with strictly positive probability, irrespective of the recruitment technology. If an equilibrium exists where $a=u$, under $R_{u}$ there is absence of unemployment, while under $R_{1}, \ldots, R_{u-1}$, unemployment does arise with positive probability.

Proof. Note that if $a=u<v$, workers always get matched with a vacancy under $R_{u}$. However if $a<u<v$, the probability that all unemployed workers only apply to $a$ firms is strictly positive. In that case a maximum of $a<v$ workers get matched with vacancies and the remaining $u-a$ workers fail to find a job. For the $R_{1}, \ldots R_{u-1}$ case there is always a positive probability that a firm looses all its candidates to rival firms. ${ }^{5}$

Proposition 2 highlights that unemployment is a natural phenomenon in our job market model, even if the number of vacancies is greater than the number of workers. This is due to the fact that workers cannot coordinate where to send their applications, which implies that some vacancies receive multiple applications from different workers, while others get a single application or no applications at all. Since firms can only hire a single worker, unemployment arises with positive probability.

\footnotetext{
${ }^{5}$ Consider for example the case where workers $1,2,3$ apply to firm $A, B, C$. There is a positive probability that all firms consider workers 1 and 3 in which case, 2 remains unmatched and one of the vacancies fails to hire a worker. This argument can be made with any number of workers and jobs. Under $R_{u-1}$, there is always a positive probability that none of the $v$ vacancies considers worker $i$.
} 
Another aspect of this result is that full employment is always associated with wage dispersion (except for the special case in which the number of vacancies is equal to the number of workers looking for a job and employers apply screening technology $R_{u}$, see Proposition 1 ). This result illustrates that it is typically the case that full employment can only be achieved at the cost of wage inequality because this increases the search intensity.

We now move to the existence and characterization of equilibria. The next result characterizes existence of a degenerate wage equilibrium where workers apply for a single vacancy. For this purpose we define the following function that gives the expected payoffs of participating in the labor market for $a=1$. It is simply equal to the "urn-ball" probability of getting a job offer times the minimum wage:

$$
\gamma_{1}(\underline{w} ; u, v)=\frac{\rho v}{u} \underline{w} .
$$

where

$$
\rho=1-\left(1-\frac{1}{v}\right)^{u}
$$

Proposition 3 Let $\gamma_{1}(\underline{w} ; u, v) \geq k \geq\left[1-\left(1-z^{d}\right)^{2}\right] \underline{w}-\gamma_{1}(\underline{w} ; u, v)$, where $1-\frac{\left(1-\left(1-\frac{1}{v}\right)^{u-1}\left(1-\frac{2}{v}\right)\right) v}{(u+1)}$ $\geq z^{d} \geq 0$. Then there exists a unique symmetric equilibrium in pure strategies where all firms post the minimum wage $\underline{w}$ and workers apply to only one of the vacancies. In equilibrium, a worker is unemployed with probability $1-\gamma_{1}(\underline{w} ; u, v) / \underline{w}$, a vacancy remains unfilled with probability $\left(1-\frac{1}{v}\right)^{u}$ and a firm's expected profit is $\pi_{i}=\left(1-\left(1-\frac{1}{v}\right)^{u}\right)(1-\underline{w})$. This holds irrespective of the screening technology.

In the proof, which is in the appendix, we show that it must be the case that $k$ is sufficiently low for workers to participate in the labor market, and sufficiently high to ensure that a worker does not find it beneficial to deviate by applying to 2,3 or more vacancies.

We now turn to the existence of wage-dispersed equilibria with multiple applications. We first note that, given a worker strategy $a \geq 2$, the number of applications a firm receives is given by a binomial distribution, $\operatorname{bin}(u, a / v)$. Since in our model every firm has only one vacancy, our wage setting game has the features of a Bertrand pricing game with capacity constrained firms. Following Dasgupta and Maskin (1986), it is easy to see that our game has a firm equilibrium in mixed strategies for given $a$. This is because $(i)$ a firm's strategy space is the closed real interval $[\underline{w}, 1],(i i)$ a firm's payoff function is continuous in $w$ except in the event that the wage the firm offers 
to a worker coincides with the wage of some other firm which considers the very same candidate and this wage happens to be the best offer the worker receives (so the set of payoff discontinuities has measure zero), and (iii) the sum of all firms' payoffs is a continuous function in a firm's strategy because a firm only steals a worker from another firm when both firms get the same profit per worker. Theorem 5 in Dasgupta and Maskin (1986) proves that an equilibrium in mixed strategies exists.

In the next section we therefore focus on providing a $3 \times 3$ example that highlights all the key mechanisms that are at work in our model. This Section illustrates that the recruitment technology is not so important for the qualitative nature of the results. In Section 5 we let the labor market get large in the usual way, i.e., $u, v \rightarrow \infty$, with $\frac{v}{u}=\theta$ and fully analyze a market where $R_{m}=R_{1}$.

\section{A market with 3 vacancies and 3 workers}

This section serves several purposes. First, it illustrates the properties of Nash equilibria in a simple environment. In particular we focus on the nature of wage dispersion and unemployment as well as on the lack of efficiency of market equilibrium. Second, it shows that different recruiting technologies lead to qualitatively similar results; this is because the economic trade-offs firms and workers face are common to both technologies. Finally, we see that these trade-offs are of different magnitude under $R_{1}$ than under $R_{3}$ and we find that there is more competition for workers under $R_{1}$, which results in higher wages and higher unemployment than under $R_{3}$.

\subsection{The $R_{3}$ recruitment technology}

Consider a market where $u=3$ and $v=3$ and $R_{m}=R_{3}$. From Lemma 1 it follows that we must examine equilibria with workers applying for 1, 2 and 3 jobs. Proposition 3 yields the equilibrium characterization when workers apply to just one job. From (1) it follows that $\gamma_{1}(\underline{w}, 3,3)=19 \underline{w} / 27$ and it is straightforward to show that a worker who deviates by sending out 2 applications has a chance of $17 / 18$ to get a job offer. Then:

Claim 1 Let $u, v=3$ and assume $\gamma_{1}(\underline{w}, 3,3) \geq k \geq \frac{17}{18} \underline{w}-\gamma_{1}(\underline{w}, 3,3)$. There exists a unique symmetric equilibrium in pure strategies where all firms post the minimum wage $\underline{w}$ and workers apply to only one of the vacancies. In equilibrium, a worker is employed with probability 19/27, a vacancy remains unfilled with probability $8 / 27$ and the expected profit for a firm is $\pi_{i}=\frac{19}{27}(1-\underline{w})$. 
Assume now that workers send multiple applications, in particular $a=2$. Then by Proposition 2 there does not exist a firm equilibrium in pure strategies. We now present the wage-dispersed equilibrium that arises in this setting. For this purpose let us define $\sigma^{n: v}$ as the expected value of the $n$-th highest wage in a random draw of size $v$ from a wage distribution $F(w)$. Moreover let

$$
\begin{aligned}
& \phi_{21}(\underline{w})=\frac{13}{81} \sigma^{1: 3}+\frac{1}{9} \sigma^{2: 3}-\frac{1}{162} \sigma^{3: 3} \\
& \phi_{23}(\underline{w})=\frac{13}{81} \sigma^{1: 3}+\frac{2}{81} \sigma^{2: 3}-\frac{7}{81} \sigma^{2: 3} .
\end{aligned}
$$

Claim 2 Let $u, v=3$ and and $\phi_{21}(\underline{w}) \geq k \geq \phi_{23}(\underline{w})$. Then, under $R_{3}$ a unique symmetric equilibrium of the game described above exists where vacancies offer wages from the set $\left[\underline{w}, \frac{21 \underline{w}+5}{26}\right]$ according to the cumulative wage distribution

$$
F(w)=\frac{4}{3}-\frac{1}{3} \sqrt{16-63 \frac{(w-\underline{w})}{(1-w)}}
$$

and workers send out 2 applications. In equilibrium workers get a job with probability $73 / 81$ and each firm receives an expected payoff of $\pi_{i}=\frac{21}{27}(1-\underline{w})$.

Proof. The payoff to a firm offering a wage $w$ when the rivals offer a random draw from $F(w)$ is

$$
\pi_{i}(w ; F(w))=\left[p^{1: 3} F^{2}(w)+2 p^{2: 3} F(w)[1-F(w)]+p^{3: 3}[1-F(w)]^{2}\right](1-w)
$$

where $p^{1: 3}, p^{2: 3}$ and $p^{3: 3}$ are the probabilities that a firm offering the highest, second highest and lowest wage in the market hires a worker, respectively. In Appendix B1 we show that $p^{1: 3}=26 / 27$, $p^{2: 3}=25 / 27$ and $p^{3: 3}=21 / 27$. Substitution of them in (5) yields:

$$
\pi_{i}(w ; F(w))=\left[-\frac{1}{9} F^{2}(w)+\frac{8}{27} F(w)+\frac{21}{27}\right](1-w)
$$

The lower bound of the support of the wage distribution $F(w)$ must be $\underline{w}$. Setting $w=\underline{w}$ yields equilibrium profits: $\pi_{i}(\underline{w})=\frac{21}{27}(1-\underline{w})$. Let $\bar{w}$ denote the upper bound of the wage distribution. In a mixed strategy equilibrium, a firm must be indifferent between offering any wage in $w \in(\underline{w}, \bar{w})$ and offering the minimum wage $\underline{w}$. Thus, $F(w)$ must solve $\pi_{i}(w ; F(w))=\pi_{i}(\underline{w})$, which yields (4). Setting $F(\bar{w})=1$ and solving for $\bar{w}$ yields the upper bound of the support of the wage distribution.

Figure 1 represents the cumulative wage distribution and the frequency of wages. It is interesting to observe that high wages are more frequent than low wages, the reason being that when workers send two applications, firms must compete intensively to hire a worker. 


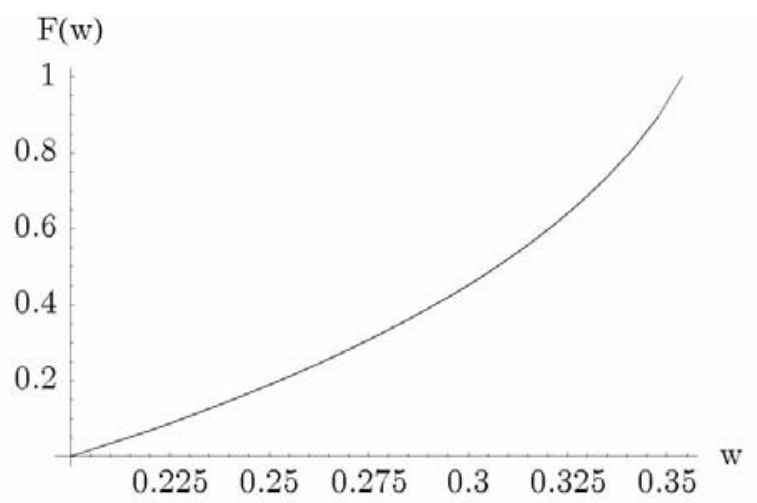

(a) Wage distribution

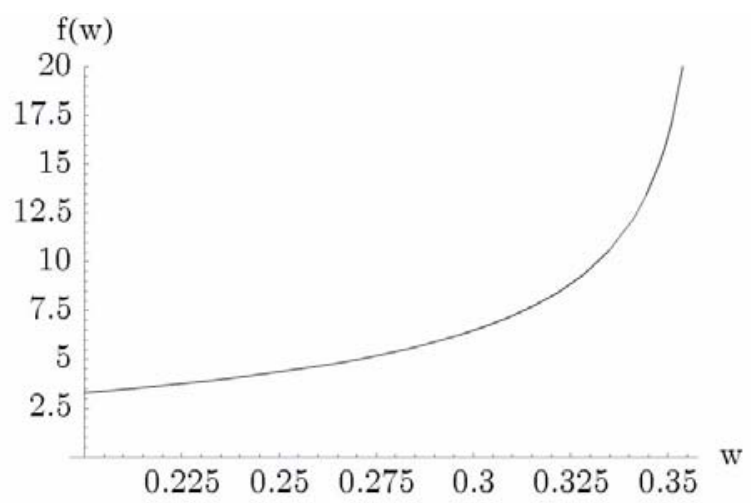

(b) Wage density

Figure 1: Equilibrium wage distribution and wage density $(a=2, \underline{w}=0.2)$

For the wage distribution (4) to be an equilibrium, we must also establish that workers have no incentives to deviate. Since a worker can be hired either for the highest, the medium, or for the minimum wage, the expected payoff for a worker when $a=2$ is given by:

$$
U^{e}(a=2)=\sigma^{1: 3} q^{1: 3}+\sigma^{2: 3} q^{2: 3}+\sigma^{3: 3} q^{3: 3}-2 k
$$

The probabilities of being hired at different wages $q^{1: 3}, q^{2: 3}$ and $q^{3: 3}$ are derived in appendix B2. Equilibrium expected gains to a worker who sends 2 applications can then be rewritten as: ${ }^{6}$

$$
U^{e}(a=2)=\frac{26}{81} \sigma^{1: 3}+\frac{25}{81} \sigma^{2: 3}+\frac{22}{81} \sigma^{3: 3}-2 k .
$$

In equilibrium, a worker must obtain non-negative expected utility, which yields the following parametric restriction:

$$
k \leq \frac{13}{81} \sigma^{1: 3}+\frac{25}{162} \sigma^{2: 3}+\frac{11}{81} \sigma^{3: 3}
$$

Let $\phi_{20}(\underline{w})$ denote the RHS of this inequality.

In addition, for $F(w)$ to be an equilibrium it must be the case that workers neither want to send a single application nor 3 applications. Consider first the case of a deviant worker who sends out 3

\footnotetext{
${ }^{6}$ We note that this expected utility can be calculated using the wage distribution (4). For this, note that $\sigma^{n: a_{l}}=\int_{-\infty}^{\infty} \frac{a_{l} !}{(n-1) !\left(a_{l}-n\right) !} w F(w)^{n-1}(1-F(w))^{a_{l}-n} f(w) d w, \quad \sigma^{1: 3}=3 \int w F^{2}(w) f(w) d w \quad, \quad \sigma^{2: 3}=$ $\int 6 w(1-F(w)) F(w) f(w) d w$ and $\sigma^{3: 3}=\int 3 w(1-F(w))^{2} f(w) d w$. Therefore, the expected utility for a worker who sends out 2 applications is:

$$
U^{e}(a=2)=\int_{\underline{w}}^{\bar{w}}\left(\frac{2}{9} F(w)-\frac{2}{27} F^{2}(w)+\frac{22}{27}\right) w f(w) d w-2 k .
$$
}


applications rather than 2. This worker secures a job but incurs higher application costs. Let $q_{3}^{1: 3}$ be the probability that the deviant gets an offer with the highest wage; likewise, let $q_{3}^{2: 3}$ and $q_{3}^{3: 3}$ be the probability that she gets the second highest wage and the lowest wage when deviating to 3 applications, respectively. In appendix B3 we derive that $q_{3}^{1: 3}=13 / 27, q_{3}^{2: 3}=1 / 3$ and $q_{3}^{3: 3}=5 / 27$. Then, the expected utility for the deviant is given by

$$
U_{d}^{e}\left(a^{d}=3\right)=\frac{13}{27} \sigma^{1: 3}+\frac{1}{3} \sigma^{2: 3}+\frac{5}{27} \sigma^{3: 3}-3 k
$$

Deviating to $a=3$ is not profitable if

$$
\begin{aligned}
U^{e}(a & =2) \geq U_{d}^{e}\left(a^{d}=3\right), \text { or: } \\
k & \geq\left(\frac{13}{27}-\frac{26}{81}\right) \sigma^{1: 3}+\left(\frac{1}{3}-\frac{25}{81}\right) \sigma^{2: 3}+\left(\frac{5}{27}-\frac{22}{81}\right) \sigma^{3: 3} \\
k & \geq \frac{13}{81} \sigma^{1: 3}+\frac{2}{81} \sigma^{2: 3}-\frac{7}{81} \sigma^{3: 3}=\phi_{23}(\underline{w})
\end{aligned}
$$

Finally, we have to establish when it is not profitable to deviate to $a=1$. Consider a worker who deviates and sends out just one application. As before, let $q_{1}^{1: 3}$ be the probability that this deviant gets the maximum wage, $q_{1}^{2: 3}$ be the probability she gets the second highest wage, and $q_{1}^{3: 3}$ be the probability to get the lowest wage. We derive in appendix B.3 that $q_{1}^{1: 3}=13 / 81, q_{1}^{2: 3}=16 / 81$ and $q^{3: 3}=15 / 54$. The expected utility for a deviant in this case is given by:

$$
U_{d}^{e}\left(a^{d}=1\right)=\frac{13}{81} \sigma^{1: 3}+\frac{16}{81} \sigma^{2: 3}+\frac{15}{54} \sigma^{3: 3}-k
$$

Deviating to $a=1$ is not profitable if:

$$
\begin{aligned}
U^{e}(a & =2) \geq U_{d}^{e}(a=1) \text { or: } \\
k & \leq\left(\frac{26}{81}-\frac{13}{81}\right) \sigma^{1: 3}+\left(\frac{25}{81}-\frac{16}{81}\right) \sigma^{2: 3}+\left(\frac{22}{81}-\frac{15}{54}\right) \sigma^{3: 3} \\
k & \leq \frac{13}{81} \sigma^{1: 3}+\frac{1}{9} \sigma^{2: 3}-\frac{1}{162} \sigma^{3: 3}=\phi_{21}(\underline{w})
\end{aligned}
$$

Note that $\phi_{21}(\underline{w}) \leq \phi_{20}(\underline{w})$; therefore if condition (7) holds, workers obtain non-negative utility. The proof is now complete.

We finally consider the case where each worker sends 3 applications. The following result characterizes equilibrium in this case. Let $\Omega(\underline{w})=\underline{w} / 3$.

Claim 3 Let $u, v=3$ and $k \leq \Omega(\underline{w})$. Then under $R_{3}$ a unique symmetric equilibrium exists where 
all vacancies offer the minimum wage $\underline{w}$ and workers send out three applications. In equilibrium workers get a job for sure and firms receive an expected payoff of $\pi_{i}=(1-\underline{w})$.

Proof. When workers apply to all 3 jobs, firms get a worker for sure and have no incentive to pay a wage above the minimum wage. The expected payoff for a worker is $U^{e}(a=3)=\underline{w}-3 k$. In equilibrium, a worker must obtain non-negative expected utility. This yields the parametric restriction $k \leq \frac{1}{3} \underline{w}=\Omega(\underline{w})$.

We now check the conditions under which workers do not deviate. Consider a worker who deviates by sending out 2 applications. The probability that this worker gets no job is $\frac{1}{3}$. This occurs when vacancies to which the deviant applies for get occupied by other workers. Hence, the expected payoff for the deviant is $U_{d}^{e}\left(a^{d}=2\right)=\frac{2}{3} \underline{w}-2 k$. Deviating to $a=2$ is not profitable if $U^{e}(a=3) \geq U_{d}^{e}\left(a^{d}=2\right)$ or $\underline{w}-3 k \geq \frac{2}{3} \underline{w}-2 k$, which is always satisfied when the non-negative expected utility condition holds. Deviating to $a=1$ is obviously a less profitable deviation when $k \leq \underline{w} / 3$. This completes the proof.

\section{Overview of equilibria for the case of 3 vacancies and 3 workers under $R_{3}$.}

As shown above, in a market with 3 vacancies and 3 workers equilibria exist where workers apply to either one, two or three vacancies. When workers apply to two vacancies, the equilibrium exhibits wage dispersion while when workers apply to either one or three jobs, all firms post the minimum wage with probability one in equilibrium. When workers apply for one or two jobs, there is unemployment while each worker gets a job for sure when they apply for three jobs.

Firm profits are increasing in the number of applications in this $3 \times 3$ example. An increase in workers search intensity has two effects. On the one hand, it increases competition between firms, which tends to reduce firms' profits. On the other hand, firms increase the probability to fill their vacancies. The second effect is of greater magnitude in this example and thus firms' profits are increasing in the number of applications sent by the workers in equilibrium. In the next section we show that this does not hold in a more general setting.

The different equilibria exist for distinct parameter constellations. In Figure 2(a) we have plotted, in the $(k, \underline{w})$ space, the functions $\gamma(\cdot), \phi_{21}(\cdot), \phi_{23}(\cdot)$ and $\Omega(\cdot)$ that appear in the Propositions above. These functions define the parameter areas for which the different equilibria exist. The shaded regions labelled " $a=1$ ", " $a=2$ " and " $a=3$ " indicate the distinct equilibria. The Figure reveals that for high costs of application, say $k_{H}$, only the equilibrium where workers apply to just 
one job exists and this requires the minimum wage to be quite high. For intermediate application costs, say $k_{M}$, if the minimum wage is high an equilibrium with $a=2$ or $a=3$ exists while if the minimum wage is moderate then a single application equilibrium exists. Finally, for low application costs, like $k_{L}$, workers typically apply to three jobs in equilibrium.

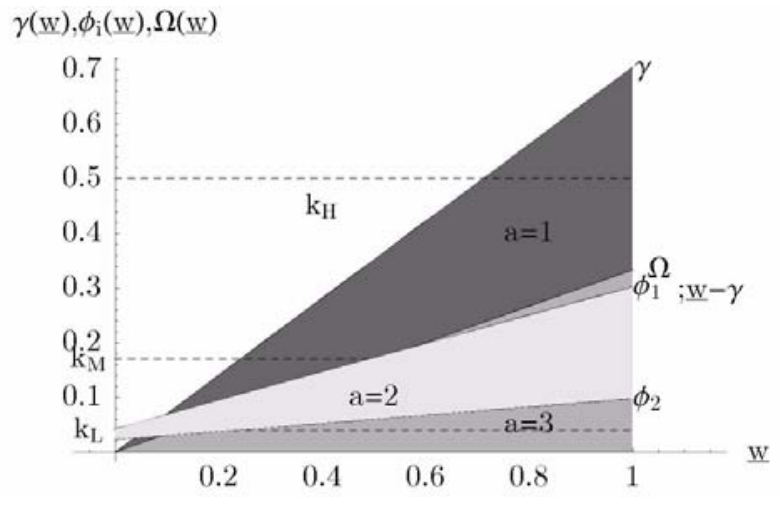

(a) Equilibria

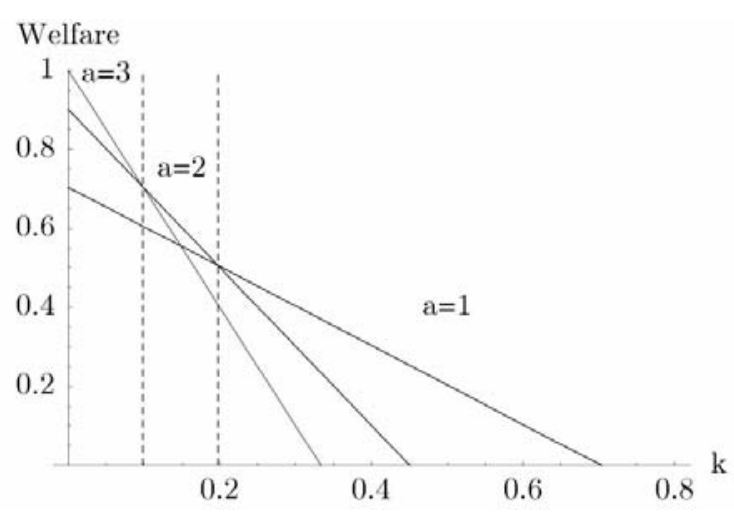

(b) Welfare

Figure 2: Equilibria and Welfare in a $3 \times 3$ model under $R_{3}$.

There are two aspects related to Figure 2(a) worth to discuss. The first refers to the effects of an increase in the minimum wage on unemployment. ${ }^{7}$ The graph reveals that unemployment is weakly decreasing in $\underline{w}$. For high application costs, an increase in the minimum wage has no impact on unemployment. By contrast, when application costs are moderate or low, unemployment decreases in $\underline{w}$. The reason for this result is that a higher minimum wage makes applying for more jobs a more attractive strategy and thus a worker's probability of being employed increases. This could explain some of the controversies of the huge empirical literature on minimum wages, see e.g. Brown (1999) for an overview. The second aspect pertains to the effects of application costs on unemployment. The Figure reveals that, for a fixed minimum wage, unemployment is typically weakly increasing in $k$. What happens is that for low application costs workers apply to all jobs for sure; for intermediate costs they apply for two jobs only and run into the risk of remaining unemployed. Finally, for high $k$ they apply for a single job and fail more frequently to get a job.

Figure 2(b) shows the levels of welfare attained under the different equilibria. The most efficient equilibrium is the one that maximizes output (number of matches times one) minus total application cost. The graph shows the regions where $a=1, a=2$ and $a=3$ are efficient respectively. The minimum wage has no direct impact on efficiency, it only redistributes income from the firm to the

\footnotetext{
${ }^{7}$ Note that only for $R_{3}$, unemployment is always decreasing in the number of applications.
} 
worker. It can however be used to select the most favorable equilibrium. A comparison of 2(a) of and 2(b) reveals that workers can send either too many or too few applications in equilibrium. For example, if $k=0.25$ Figure 2(b) tells us that it is efficient that workers apply for a single job; in that case Figure 2(a) shows that the minimum wage should be set sufficiently high so that workers do participate in the labor market but not too high so that workers apply to multiple jobs. Similarly, if $k$ is close to zero, the minimum wage should be set sufficiently high to select the $a=3$ equilibrium.

\section{Wages and labor market tightness.}

We are interested in the relationship between wage setting and the vacancy-to-unemployment ratio $v / u$. Equilibrium wage distributions for different markets when workers apply for two jobs $(a=2)$ are compared in Figure 3 below. ${ }^{8}$ Interestingly the wage distributions can be ranked according to the first order stochastic dominance criterion. As we increase the $v / u$ ratio, firms compete more intensively for workers and offer higher wages. In Section 5 we shall show that this result also arises in "large" labor markets.

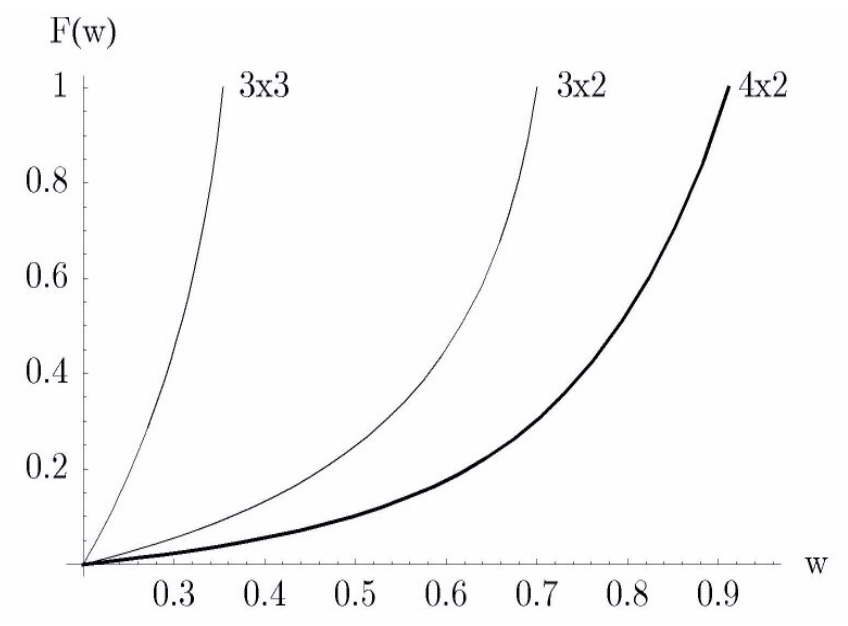

Figure 3: Wage distributions for $v \times u=3 \times 3,3 \times 2$ and $4 \times 2(\underline{w}=0.2, a=2)$.

\subsection{The $R_{1}$ recruitment technology}

In this Section we derive equilibria in the $3 \times 3$ labor market model when firms can offer the job only to one candidate. Under $R_{1}$ there will be fewer matches in equilibrium than under $R_{3}$, so we

\footnotetext{
${ }^{8}$ The derivations for the $3 \times 2$ and $4 \times 2$ are similar and can be obtained from the authors upon request.
} 
can think of this case as a high frictions case. Interestingly, workers can actually be better off in this high frictions case because firms compete more intensively for them and the resulting wage distribution (first order) stochastically dominates the one of the low frictions case. Most derivations are similar to the ones under $R_{3}$, so we only summarize for which values of $k, a=1, a=2$, and $a=3$ are equilibria and what the associated wage distributions are (details of the derivations can be found in the Appendix).

For obvious reasons, if workers send just one application, the possibility of second round offers has no bearing on wage setting behavior. Hence Claim 1 also holds here.

Assume now that workers send out 2 applications each. In this case, it is convenient to introduce the following notation: let $E_{n}$ denote the expected maximum wage from a random sample of size $n$ from the wage distribution $F(w)$ and let

$$
\begin{aligned}
& \phi_{20}(\underline{w})=\frac{1}{2}\left(\frac{86}{162} E_{1}+\frac{35}{162} E_{2}\right) \\
& \phi_{21}(\underline{w})=-\frac{17}{162} E_{1}+\frac{25}{162} E_{2}+\frac{15}{162} E_{3}
\end{aligned}
$$

Claim 4 Let $u, v=3$ and $\phi_{20}(\underline{w}) \geq k \geq \phi_{21}(\underline{w})$. Then under $R_{1}$ a unique symmetric equilibrium exists where workers send out 2 applications and firms choose wages from the set $\left[\underline{w}, \frac{35+43 \underline{w}}{78}\right]$ according to the cumulative wage distribution

$$
F(w)=\frac{43}{35} \frac{(w-\underline{w})}{(1-w)}
$$

In equilibrium workers get a job with probability $\frac{121}{162}$ and firms obtain an expected payoff of $\pi_{i}=$ $\frac{43}{81}(1-\underline{w})$.

It is interesting to compare equilibrium wage setting with and without second round offers for this case where $a=2$. Figure 4 represents wage setting under $R_{3}$, given in (4), and under $R_{1}$ (equation (8)). The graph shows that (8) dominates (4) under the criterion of first-order stochastic dominance. This implies that firm competitiveness is enhanced by the absence of second round offers and thus worker expected utility may be greater in the more frictional case. For the intermediate case $R_{2}$ one would expect the wage distribution to lie in between the two curves.

We now present the results in case workers send 3 applications. For this purpose let

$$
\phi_{32}(\underline{w})=\frac{1}{9} E_{2}+\frac{1}{27} E_{3}
$$




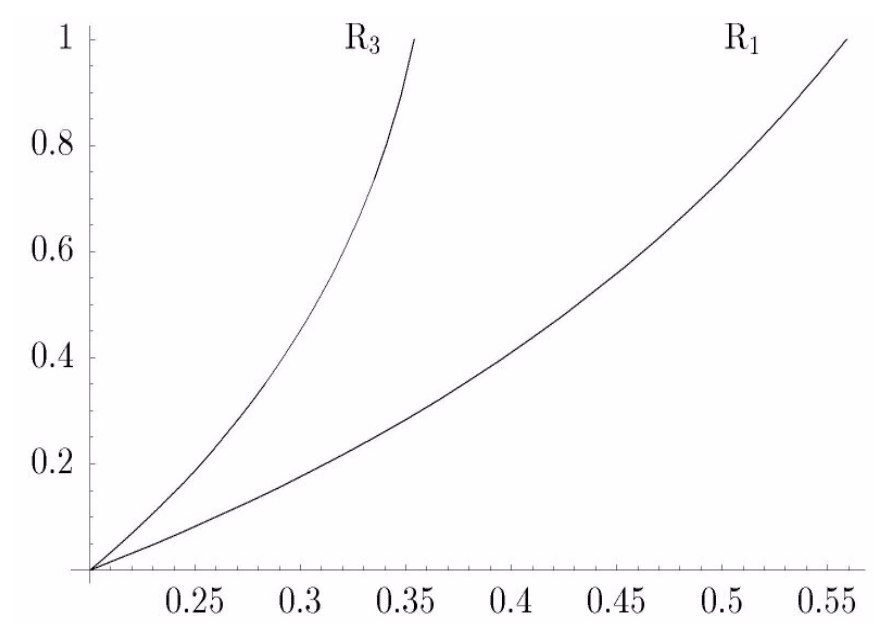

Figure 4: Wage distributions under $R_{u}$ and $R_{1}(3 \times 3$ market, $\underline{w}=0.2, a=2)$.

Claim 5 Let $u, v=3$ and $\phi_{32}(\underline{w}) \geq k$. Then under $R_{1}$ a unique symmetric equilibrium exists where workers send out 3 applications and firms choose wages from the set $\left[\underline{w}, \frac{5+4 \underline{w}}{9}\right]$ according to the cumulative wage distribution

$$
F(w)=2\left(\sqrt{\frac{1-\underline{w}}{1-w}}-1\right)
$$

In equilibrium workers get a job with probability $\frac{19}{27}$ and firms obtain an expected payoff of $\pi_{i}=$ $\frac{4}{9}(1-\underline{w})$.

The difference with section 4.1 where firms could make second and third round offers is that now a firm can fail to hire a worker and therefore there is competition for workers. This results in wage dispersion and, interestingly, may result in greater worker welfare. To illustrate this, consider the case that $\underline{w}=3 k$; with second round offers, firms offer the minimum wage in equilibrium and the payoff to a worker is thus zero. By contrast, in the case without second round offers there is wage dispersion (for a low $\underline{w}$ a $a=3$ equilibrium exists) and thus a worker's expected payoff is strictly positive. Hence, a more restrictive matching technology can increase the expected utility of workers by making them effectively scarcer.

The set of parameters for which distinct equilibria exist under the recruitment technology $R_{1}$ is represented in Figure 5(a).

A comparison of Figures 2(a) and 5(a) reveals that the screening technology does not affect the 


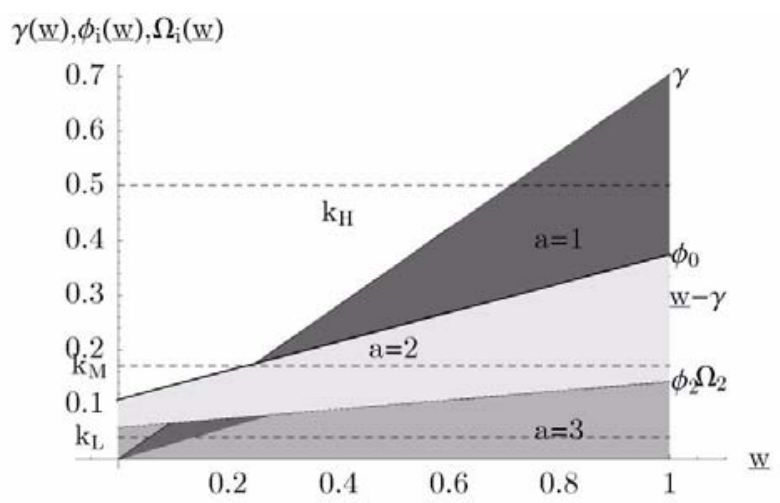

(a) Equilibria

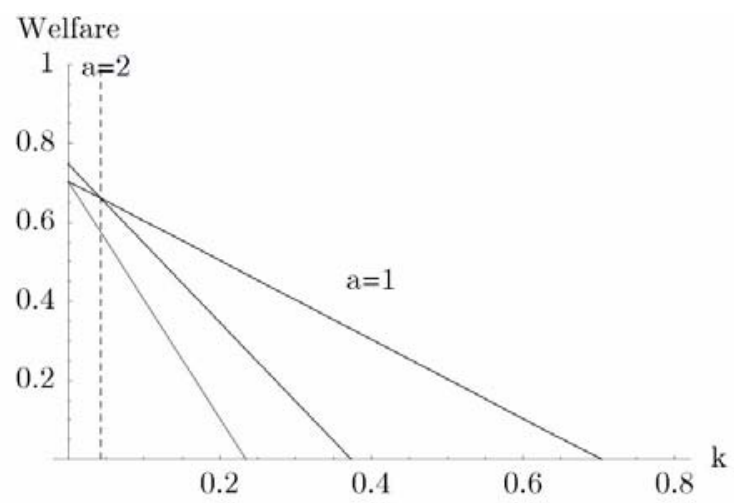

(b) Welfare

Figure 5: Equilibria and Welfare in a 3x3 model under $R_{1}$.

qualitative observations we have made above. $R_{1}$ has the advantage that the combinatorics needed to calculate expected payoffs for firms and workers is easier for large labor markets. In what follows we will confine the analysis to $R_{1}$ and derive general properties of large labor markets.

Figure 5(b) plots aggregate welfare for the $R_{1}$ case for different values of $k$. The main difference with the $R_{3}$ case in Figure 2(b) is that $a=3$ is never efficient. The reason is that $a=1$ and $a=3$ generate the same number of matches because under $a=1$ all three workers apply to one job while the $a=3$ case can be thought of as all three firms applying to one worker. Since applying is costly, $a=3$ can never be efficient and the minimum wage should be set low so that it prevents workers from sending an excessive number of applications.

\section{$5 \quad$ Large labor markets}

In this section we let the labor market get large in the usual way, i.e., $u, v \rightarrow \infty$, with $\frac{v}{u}=\theta$. We restrict ourselves to the $R_{1}$ case as argued above. We focus on existence and characterization of the equilibrium with multiple applications (the equilibrium for $a=1$ follows straightforwardly from Proposition 3). We are also interested in how the equilibrium number of applications and the wage distribution are affected by the market tightness parameter $\theta$. Later in this Section we endogenize $\theta$ by examining the free-entry long-run equilibrium and we study the efficiency properties of the equilibrium.

Our first result characterizes wage setting behavior by firms, given workers strategies. 
Proposition 4 For any value of $\theta$, and $a \geq 2$, there exists an equilibrium wage distribution

$$
F(w ; a, \theta, \underline{w})=\frac{1-z(\theta, a)}{z(\theta, a)}\left[\left[\frac{1-\underline{w}}{1-w}\right]^{\frac{1}{a-1}}-1\right]
$$

with support $\left[\underline{w}, 1-(1-\underline{w})(1-z(\theta, a))^{a-1}\right]$, where $z(\theta, a)=\frac{\left(1-\exp \left(-\frac{a}{\theta}\right)\right) \theta}{a}$. Further, the distribution of wages $F(w ; a, \theta, \underline{w}+\triangle)$ dominates in a first-order stochastic sense the distribution of wages $F(w ; a, \theta, \underline{w})$, and $F(w ; a, \theta+\triangle, \underline{w})$ first-order stochastically dominates $F(w ; a, \theta, \underline{w})$.

Proof: Recall that $\rho(u, v, a)$ is the ("urn ball") probability that a firm gets at least one applicant. Thus:

$$
\rho(\theta, a)=\lim _{u, v \rightarrow \infty, \frac{v}{u}=\theta}\left(1-\left(1-\frac{a}{v}\right)^{u}\right)=1-\exp \left(-\frac{a}{\theta}\right)
$$

If the firm receives more than one application, the firm picks one of them at random. The (randomly chosen) applicant has sent a total of $a$ applications, so he can potentially get $1,2,3, \ldots$, up to a total of $a-1$ other wage offers.

Let $z(u, v, a)$ be the probability for one of the $(a-1)$ other applications of the worker to result in a job offer. We use the result of Albrecht et al. (2003) that in the limit $\left(u, v \rightarrow \infty, \frac{v}{u}=\theta\right)$, getting an offer from firm $j$ is independent of getting an offer from firm $i \neq j$. Then, $z(\theta, a)=$ $\lim _{u, v \rightarrow \infty, \frac{v}{u}=\theta} z(u, v, a)$ is simply equal to the number of firms with applications divided by the total number of applications:

$$
z(\theta, a)=\frac{\rho(\theta, a) \theta}{a}=\frac{\left(1-\exp \left(-\frac{a}{\theta}\right)\right) \theta}{a}
$$

A firm does not compete for the worker in question with probability $(1-z(\theta, a))^{a-1}$, competes with just one other firm with probability $(a-1) z(\theta, a)(1-z(\theta, a))^{a-2}$, and so on. Thus the expected payoff to a firm $i$ can be written as:

$$
\pi^{i}(w ; F(\cdot))=\rho(\theta, a)\left(\sum_{j=0}^{a-1}\left(\begin{array}{c}
a-1 \\
j
\end{array}\right) z(\theta, a)^{j}(1-z(\theta, a))^{a-1-j} F(w)^{j}\right)(1-w)
$$

We can use the binomial theorem to simplify (10) to:

$$
E \pi^{i}(w ; F(\cdot))=\rho(\theta, a)[1-z(\theta, a)+z(\theta, a) F(w)]^{a-1}(1-w)
$$

Since in a mixed strategy equilibrium, all firms must be indifferent between strategies, profits 
of any firm must be equal to the profits of a firm that offers the minimum wage.

$$
E \pi^{i}(\underline{w} ; F(\cdot))=\rho(\theta, a)(1-z(\theta, a))^{a-1}(1-\underline{w})
$$

Solving $E \pi(w ; F(\cdot))=E \pi(\underline{w} ; F(\cdot))$ yields the equilibrium wage distribution given above. The upper bound of the support of the wage distribution can be found by setting $F(\bar{w}, a, \theta)=1$. It is easy to see that the upper bound of the distribution of wages is less than 1, increasing in $a$ (for a given $\theta$ ) and in $\theta$ (for a given $a$ ).

To prove the results on stochastic dominance, we first note that $F(\cdot)$ is monotonically decreasing in $z(\theta, a)$ and in $\underline{w}$. Secondly we note that the function $z(\theta, a)$ is increasing in $\theta$, which can be seen from

$$
\frac{d z(\theta, a)}{d \theta}=\frac{\theta \exp \left(\frac{a}{\theta}\right)-(a+\theta)}{a \theta \exp \left(\frac{a}{\theta}\right)}
$$

Notice that the LHS of the numerator increases exponentially in $a$ while the RHS increases linearly; thus, if $d z(\theta, a) / d \theta>0$ for $a=2$, then the same inequality holds generally. We then need to check that $\theta(\exp (2 / \theta)-1)>2$, which holds for all $\theta>0$. The proof is now complete.

Proposition 4 shows that an increase in the vacancy-to-unemployment ratio results in a rightward shift of the wage distribution. Thus, wage setting behavior becomes more competitive as the market tightness parameter $\theta$ increases. An increase in the minimum wage $\underline{w}$ has a similar influence on the wage distribution.

We now discuss how the average wage depends on the number of applications $a$. Using Proposition 4 , the expected wage can be written as (using integration by parts and changing the variable of integration)

$$
E[w ; \theta, a]=1-\int_{0}^{1} \frac{(1-\underline{w})(1-z(\theta, a))^{a-1}}{(1-z(\theta, a)(1-y))^{a-1}} d y .
$$

The influence of the number of applications on the average wage is given by the following expression:

$$
\frac{d E[w]}{d a}=\frac{\partial E[w]}{\partial a}+\frac{\partial E[w]}{\partial z} \frac{d z}{d a} .
$$

We note that

$$
\begin{gathered}
\frac{\partial E[w]}{\partial z}=\int_{0}^{1} \frac{(1-\underline{w})(a-1) y(1-z)^{a-2}}{(1-z(1-y))^{a}}>0 \\
\frac{d z}{d a}=\frac{1-z}{a}-\frac{z}{\theta}<0
\end{gathered}
$$


while

$$
\frac{\partial E[w]}{\partial a}=-\int_{0}^{1} \frac{(1-\underline{w})(1-z)^{a-1}}{(1-z(1-y))^{a-1}} \ln \left[\frac{1-z}{1-z(1-y)}\right] d y>0
$$

where the last inequality follows from the fact that the integrand is negative. From the sign of these partial derivatives, it follows that an increase in $a$ results in two forces on competitiveness that go in opposite directions. To understand this, consider a firm offering a job to a candidate when each worker sends out $a$ applications. If the worker increases the number of applications to $a+1$, one force increases the probability that the firm looses the worker to another firm simply because the worker can get one additional offer with a higher wage. This effect increases firm competitiveness and is captured by the first term of the RHS of equation (13). However, when all workers send $a+1$ applications, the probability that each of the other $a$ applications of the candidate in question results in a job offer falls. This is simply due to the fact that all firms have more applicants to choose from. This effect tends to weaken firms competition and is captured by the second term in the RHS of (13). Which effect dominates turns out to be sensitive to market parameters but we can prove the following result:

Corollary 1 There exists a sufficiently large $\widetilde{\theta}$ such that the expected wage increases in the number of applications a for all $\theta \geq \widetilde{\theta}$. Further, in a neighborhood of $a=2$, the expected wage increases in a for all $\theta$.

Corollary 1 shows two results. First, that the expected wage is always increasing in the number of applications in a neighborhood of $a=2$. The idea here is that the second force $(d z / d a<0)$ is small when the number of applications is low. In that case, competition unambiguously increases when workers send more applications. The second result is that the expected wage is always increasing in the number of applications provided that there are sufficiently many firms around. The idea is similar. When there are many firms in the market, an increase in the number of applications $a$ is likely to increase the number of firms that compete for the same worker, which increases firm competitiveness. Numerical analysis of equation (13), however, has revealed that the expected wage may fall in $a$ for some parameters; this can be seen in Figure 6 where we have computed the expected wage for different values of the labor market tightness parameter $\theta$. The Figure shows that when $a$ is relatively large (e.g. $10<a<80$ ) and $2<\theta<4$ then the expected wage decreases in the number of applications workers send out. The graph also illustrates the stochastic dominance result provided in Proposition 4: for a fixed $a$, the expected wage increases in $\theta$. 


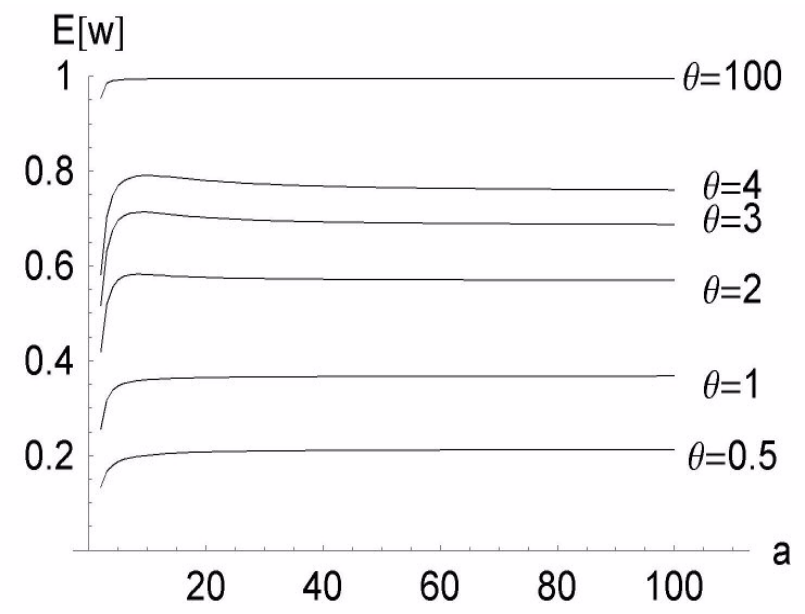

Figure 6: Expected wage and the number of applications (for different $\theta$ values, $\underline{w}=0$ ).

We now turn to the workers problem. A worker maximizes utility taking firm wage setting and other workers strategies as given. The (gross) expected utility of a worker sending $a_{l}$ applications when the rest of the workers sends $a$ applications is given by the following expression:

$$
E u\left(a_{l} ; a\right)=\int_{\underline{w}}^{\bar{w}}\left(\sum_{j=1}^{a_{l}}\left(\begin{array}{c}
a_{l} \\
j
\end{array}\right) z(\theta, a)^{j}(1-z(\theta, a))^{a_{l}-j} \cdot j \cdot F(w)^{j-1}\right) w f(w) d w
$$

This expression tells us that a worker who sends $a_{l}$ applications gets a single offer with probability $a_{l} z(1-z)^{a_{l}-1}$ and in this case the wage the worker expects to get is the mean wage; the worker gets two offers with probability $\left(\begin{array}{c}a_{l} \\ 2\end{array}\right) z^{2}(1-z)^{a_{l}-2}$ and then the wage the worker expects to get is the maximum of a random sample of size 2 ; and so on and so forth. ${ }^{9}$ Using the binomial theorem, equation (16) can be rewritten as

$$
E u\left(a_{l} ; a\right)=a_{l} z(\theta, a) \int_{\underline{w}}^{\bar{w}}[1-z(\theta, a)(1-F(w))]^{a_{l}-1} w f(w) d w
$$

and integration by parts yields:

$$
E u\left(a_{l} ; a\right)=\bar{w}-\underline{w}(1-z(\theta, a))^{a_{l}} \int_{\underline{w}}^{\bar{w}}[1-z(\theta, a)(1-F(w))]^{a_{l}} d w
$$

\footnotetext{
${ }^{9}$ In the empirical job search literature an important distinction is made between the offer distribution, $F$ and the distribution of accepted wage offers which is in our case very similar to the term in brackets of $(16): G(a, \theta, w)=$ $\sum_{j=0}^{a-1}\left(\begin{array}{c}a-1 \\ j\end{array}\right) z(\theta, a)^{j}(1-z(\theta, a))^{a-1-j} \cdot F(w)^{j}$. This is basically a combination of the wage offer $F(w)$ and the probability that the worker accepts the offer which depends on the number of other offers and on the magnitude of the offer.
} 
Changing the variable of integration appropriately, we can rewrite a typical worker's utility as:

$$
E u\left(a_{l} ; a\right)=\int_{(1-z(\theta, a))^{a_{l}}}^{1}\left(1-\frac{(1-\underline{w})(1-z(\theta, a))^{a_{l}-1}}{y^{\frac{a_{l}-1}{a_{l}}}}\right) d y
$$

Integration of this expression yields:

$$
E u\left(a_{l} ; a\right)=1-(1-z(\theta, a))^{a_{l}}-\frac{a_{l}(1-\underline{w})(1-z(\theta, a))^{a-1}\left(1-(1-z(\theta, a))^{a_{l}+1-a}\right)}{a_{l}+1-a}
$$

The marginal gains to a worker sending $a_{l}$ applications are given by

$$
\begin{aligned}
\frac{d E u\left(a_{l} ; a\right)}{d a_{l}} & =-(1-z(\theta, a))^{a_{l}} \log [1-z(\theta, a)]+\frac{(1-\underline{w})(1-z(\theta, a))^{a-1}}{\left(a_{l}+1-a\right)^{2}} \\
& {\left[(a-1)\left(1-(1-z(\theta, a))^{a_{l}+1-a}\right)+a_{l}\left(a_{l}+1-a\right) \log [1-z(\theta, a)](1-z(\theta, a))^{a_{l}+1-a}\right] }
\end{aligned}
$$

In symmetric equilibrium, the marginal gains from applying to $a$ vacancies must be equal to the marginal cost $k$. Invoking symmetry in (18) yields the following equilibrium condition:

$$
\left|\frac{d E u\left(a_{l} ; a\right)}{d a_{l}}\right|_{a_{l}=a}=k
$$

Or

$-(1-z(\theta, a))^{a} \log [1-z(\theta, a)]+(1-\underline{w})(1-z(\theta, a))[(a-1) z(\theta, a)+a(1-z(\theta, a)) \log [1-z(\theta, a)]=k$

The LHS of (20) represents the equilibrium marginal gains to workers when they apply to $a$ vacancies. Let us denote these marginal gains as $\Psi(a ; \theta, \underline{w})$. We now make the following observations on this function: (i) $\Psi(a ; \theta, \underline{w})$ is continuous in $a$ and strictly positive everywhere. The latter follows from noting that

$$
\begin{aligned}
\Psi(a, \theta, \underline{w}) & >-(1-\underline{w})(1-z)^{a} \log [1-z]+(1-\underline{w})(1-z)[(a-1) z+a(1-z) \log [1-z] \\
& =(1-\underline{w})(1-z)^{a-1}(a-1)[z+(1-z) \log (1-z)]>0
\end{aligned}
$$

where the last inequality follows from $z \in(0,1)$. (ii) It is easy to see that these marginal gains converge to zero as $a$ approaches infinity. These two conditions guarantee existence of equilibrium for sufficiently low application cost $k \cdot{ }^{10}$ As a result, we can state:

\footnotetext{
${ }^{10}$ To show uniqueness we need to check that, for any $\theta$ and $\underline{w}, d \Psi(a) / d a<0$. Analytically this is difficult but numerical analysis of this derivative suggests that this is the case.
} 
Theorem 1 Let $0<k \leq \Psi(2, \theta, \underline{w})$. Then an equilibrium exists where workers send $a^{*}(\geq 2)$ applications and firms randomly choose wages from the set $\left[\underline{w}, 1-(1-\underline{w})\left(1-z\left(\theta, a^{*}\right)\right)^{a^{*}-1}\right]$ according to the wage distribution

$$
F\left(w ; a^{*}, \theta, \underline{w}\right)=\frac{1-z\left(\theta, a^{*}\right)}{z\left(\theta, a^{*}\right)}\left[\left[\frac{1-\underline{w}}{1-w}\right]^{\frac{1}{a^{*}-1}}-1\right]
$$

where $a^{*}$ solves $\Psi\left(a^{*} ; \theta, \underline{w}\right)-k=0$. The equilibrium unemployment rate is $\left(1-z\left(\theta, a^{*}\right)\right)^{a^{*}}$.

Our next question is how an increase in the vacancy-to-unemployment ratio, $\theta$, affects the equilibrium number of applications $a^{*}$ as well as unemployment rate. To answer this question, we examine how the equilibrium marginal gains from applications change with $\theta .{ }^{11}$ This follows from the following derivative:

$$
\frac{d \Psi(a, \theta, \underline{w})}{d \theta}=\frac{-(a-1)\left(\theta\left(e^{\frac{a}{\theta}}-1\right)-a\right)(1-z)^{a}}{\theta\left(a e^{\frac{a}{\theta}}-\theta\left(e^{\frac{a}{\theta}}-1\right)\right)^{2}}\left[(a-1) \theta\left(e^{\frac{a}{\theta}}-1\right)+a\left(a e^{\frac{a}{\theta}}-\theta\left(e^{\frac{a}{\theta}}-1\right)\right) \log [1-z]\right)
$$

The sign of the first factor in (21) is negative since $\theta\left(e^{\frac{a}{\theta}}-1\right)-a$ is always positive. Thus, the sign of this derivative depends only on the last factor in the numerator. Let $\Upsilon(\theta, a)=(a-1) \theta\left(e^{\frac{a}{\theta}}-\right.$ $1)+a\left(a e^{\frac{a}{\theta}}-\theta\left(e^{\frac{a}{\theta}}-1\right)\right) \log [1-z]$. We note that for any given $a, z(\theta, a)$ is continuous in $\theta$, converges to 1 as $\theta$ approaches infinity and converges to zero as $\theta$ approaches zero. As a result, $\Upsilon(\theta, a)$ is negative for $\theta$ small and positive for $\theta$ large. This implies that:

Proposition 5 There exists a sufficiently small $\underline{\theta}$ such that for all $\theta<\underline{\theta}$ the equilibrium number of applications $a^{*}$ is increasing in $\theta$. Moreover, there exists a sufficiently large $\bar{\theta}$ such that for all $\theta>\bar{\theta}$ the equilibrium number of applications $a^{*}$ is decreasing in $\theta$.

This result shows that the equilibrium number of applications is non-monotonic in $\theta$. To our knowledge this result is new. For example, Pissarides (2000) treats search intensity as a technology parameter and finds that search effort is always increasing in $\theta$. In our model, the marginal gains from applying are decreasing in $\theta$ when $\theta$ is initially large. We illustrate this Proposition in Figure 7, where we have computed the equilibrium number of applications as a function of the market tightness parameter $\theta$, for different values of the minimum wage. The graph shows that the function $a^{*}(\theta)$ is unimodular.

\footnotetext{
${ }^{11}$ We set $\underline{w}=0$ here for simplicity.
} 


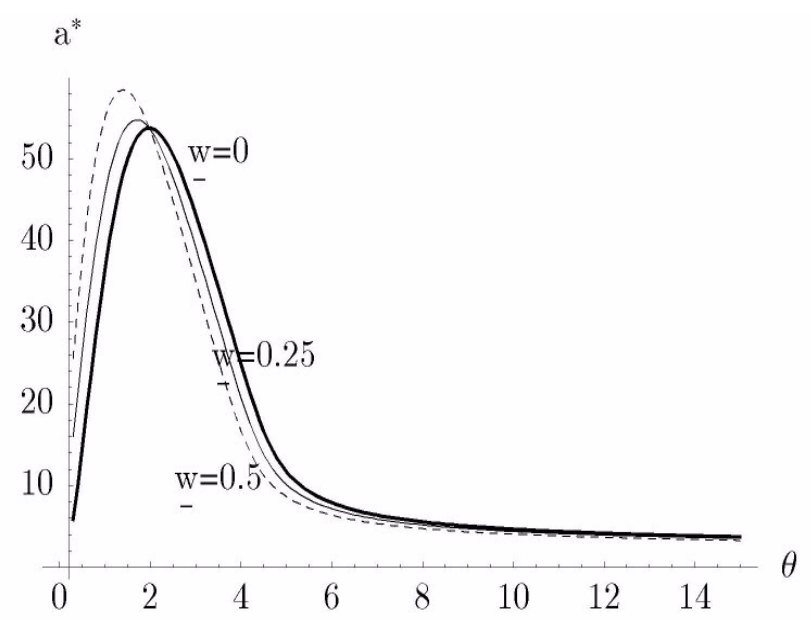

Figure 7: Equilibrium number of applications and market tightness parameter $\theta$.

The intuition is the following. We note that, for a fixed number of applications $a$, a change in $\theta$ has an effect on the probability that a worker is hired as well as on the wage the worker expects to be paid. When $\theta$ is small $(\theta \rightarrow 0)$ the total number of applications swamps the total number of vacancies. The probability that a given worker gets a job offer is quite low. A given firm receives many applicants and the likelihood that it competes with another firm for a given candidate is close to zero; as a result the wage distribution is "close" to the degenerate distribution at the minimum wage. As $\theta$ increases, the probability a worker receives a job offer goes up, and also the probability that firms have to compete for the same candidate rises; these two effects increase substantially the marginal gains from sending applications. As $\theta$ increases further, those two effects become weaker and weaker because the probability to get several job offers and the gains associated to choosing from among several wages are already large and do not increase much further. Indeed for $\theta$ relatively large each vacancy gets either no or one applicant, so the chance a firm is competing with some other firm for the same candidate is close to 1 ; as a result the wage distribution is close to the degenerate distribution at the competitive wage. Thus as $\theta$ increases beyond some critical level, the marginal gains from sending applications become smaller.

We now ask how the equilibrium unemployment rate changes with the vacancy-to-unemployment ratio. There are two effects at work here. For a given number of applications, an increase in $\theta$ increases the chance that a worker gets an offer since applications are spread over a larger set or vacancies. This effect tends to decrease the unemployment rate. However, an increase in the 
number of vacancies also changes the incentives workers have to apply for jobs (cf. Proposition 5). An analytical answer to this question thus requires to examine the following derivative:

$$
\frac{d\left(\left(1-z\left(\theta, a^{*}\right)\right)^{a^{*}}\right)}{d \theta}=\frac{\partial\left(\left(1-z\left(\theta, a^{*}\right)\right)^{a^{*}}\right)}{\partial \theta}+\frac{\partial\left(\left(1-z\left(\theta, a^{*}\right)\right)^{a^{*}}\right)}{\partial a^{*}} \frac{\partial a^{*}}{\partial \theta} .
$$

The first term of the RHS of this equation is clearly negative. Some calculations show that

$$
\frac{\partial\left(\left(1-z\left(\theta, a^{*}\right)\right)^{a^{*}}\right)}{\partial a^{*}}=(1-z)^{a^{*}}\left(\log [1-z]-1+\frac{a^{*} z}{\theta(1-z)}\right) .
$$

This expression is positive when $\theta$ is small. For large $\theta$ it depends on $a$; if $a$ is relatively small then it is negative while if $a$ is large it is positive. As a result, this derivative does not yield clear-cut results. For the majority of the parameters, the two effects mentioned above work in opposite directions. To gain some insight into this issue, we have numerically computed the probability to remain unemployed as a function of the vacancy-to-unemployment ratio. The results show a negative relationship between these two variables (see Figure 8 below).

Our last question in this section is how the minimum wage affects the equilibrium number of applications $a^{*}$ and the unemployment rate. We first examine how the marginal gains from applications change when the minimum wage goes up. This is given by the following derivative:

$$
\frac{d \Psi(a, \theta, \underline{w})}{d \underline{w}}=-(1-z(\theta, a))^{a-1}[(a-1) z(\theta, a)+a(1-z(\theta, a)) \log [1-z(\theta, a)]]
$$

We now make two remarks about this expression. One, for a fixed $a$, expression (22) converges to zero as $\theta$ approaches zero and is positive and increasing in $\theta$ in a neighborhood of $\theta=0$. Thus, for a sufficiently small $\theta$ the number of applications workers send in equilibrium is increasing in the minimum wage. Two, we note that (22) is negative for large $\theta$ and goes to zero when $\theta$ approaches infinity. Then for large $\theta$, the equilibrium number of applications is decreasing in the minimum wage.

To examine the implications of the minimum wage on unemployment rate, we compute

$$
\frac{d\left(\left(1-z\left(\theta, a^{*}\right)\right)^{a^{*}}\right)}{d \underline{w}}=\frac{\partial\left(\left(1-z\left(\theta, a^{*}\right)\right)^{a^{*}}\right)}{\partial a^{*}} \frac{\partial a^{*}}{\partial \underline{w}}
$$

As argued above, the first term of the RHS of this expression is positive when $\theta$ is small. As a result, we conclude that an increase in the minimum wage raises unemployment whenever $\theta$ is low enough. When $\theta$ is large and $a$ moderate, this term is negative and thus a minimum wage increase can decrease unemployment. Finally, when $\theta$ and $a$ are both large this term is positive and then 
again an increase in $\underline{w}$ leads to lower unemployment. The following Proposition summarizes:

Proposition 6 There exists a sufficiently small $\underline{\theta}$ such that for all $\theta<\underline{\theta}$ the equilibrium number of applications $a^{*}$ is increasing in the minimum wage $\underline{w}$. Moreover, there exists a sufficiently large $\bar{\theta}$ such that for all $\theta>\bar{\theta}$ the equilibrium number of applications $a^{*}$ is decreasing in $\underline{w}$. Moreover, the unemployment rate is increasing in $\underline{w}$ for sufficiently low $\theta$, and for sufficiently high $\theta$, while it can decrease for intermediate values of $\theta$.

The intuition behind this result is the following. First, note that an increase in the minimum wage does not affect the probability that a worker is hired but it does affect the wage a worker expects to get. When $\theta$ is low, the wage distribution is close to the degenerate distribution at the minimum wage and thus an increase in the minimum wage shifts the marginal gains from applications upwards and makes the distribution less compressed (note from Theorem 1 that the maximum wage rises when the minimum wage increases). By contrast, when $\theta$ is large, the wage distribution is close to the degenerate distribution at the competitive wage and thus an increase in the minimum wage reduces the marginal gains from applications by making the distribution even more compressed. ${ }^{12}$ The result in Proposition 6 is illustrated above in Figure 7. The graph shows that when $\theta$ is low, workers react to an increase in the minimum wage by sending more applications, while the opposite holds for $\theta$ relatively large.

The unemployment effects of an increase in the minimum wage also depend on initial conditions. For $\theta$ low (approximately $\theta \leq 1 / 2$ ), the unemployment rate is monotonically increasing in $a$ (see Albrecht et al., 2003) and we know from Proposition 6 that an increase in the minimum wage raises the equilibrium number of applications. As a result the minimum wage increases unemployment. Likewise, when $\theta>1 / 2$ unemployment is first increasing and then decreasing in $a$. We know from Proposition 5 that $a^{*}$ is excessive. Then, increasing the minimum wage compresses the wage distribution and reduces the equilibrium number of applications which also increases employment. What is striking here is that a minimum wage can be desirable because it reduces rather than increases the number of applications. This is a very different mechanism than the ones that are currently emphasized in the literature; making it more likely that a job is accepted (i.e. Burdett and Mortensen, 1998), and increasing the search intensity or driving out less productive firms (i.e.

\footnotetext{
${ }^{12}$ Lee (1999) and Teulings (2003) give empirical evidence that minimum wage increases compress the wage distribution.
} 
Van den Berg 2003). What is also striking is that a minimum wage is most desired when $\theta$ is large. In section 5.2, we will further discuss the welfare effects of the minimum wage with endogenous vacancy supply.

\subsection{Efficiency}

Is the labor market equilibrium constrained-efficient? In order to answer this question we define the planner's objective function as total output net of application cost and vacancy cost, where total output is simply equal to the number of matches times 1 . Dividing by unemployment gives the welfare per worker:

$$
\Lambda=\max _{a, \theta}\left[1-(1-z(\theta, a))^{a}\right]-a k
$$

We first examine whether workers incentives to apply for jobs are socially optimal. The first order condition with respect to $a$ is

$$
\frac{d \Lambda}{d a}=-(1-z(\theta, a))^{a-1}\left[(1-z(\theta, a)) \log [1-z(\theta, a)]-a \frac{d z(\theta, a)}{d a}\right]-k=0 .
$$

Substituting in (15) yields

$$
-(1-z(\theta, a))^{a} \log [1-z(\theta, a)]-(1-z(\theta, a))^{a-1}\left(1-z(\theta, a)-\frac{a z(\theta, a)}{\theta}\right)=k
$$

A comparison of the workers equilibrium condition (20) with (24) yields the following result:

Proposition 7 The equilibrium number of applications is excessive from the point of view of social welfare maximization.

Proof. The first term in (20) is identical to the first term in (24) so we need to show that

$$
(1-\underline{w})((a-1) z+a(1-z) \log [1-z])>1-z(\theta, a)-\frac{a z(\theta, a)}{\theta}
$$

We first note that the RHS of (25) is negative. Second, the LHS of (25) is always positive for $\theta$ large $(\theta>2.81)$ and therefore the result follows. For $\theta$ small $(\theta<2.81)$ the LHS is negative when $a$ is sufficiently small and thus we need to evaluate (25). Note that if the results holds for $\underline{w}=0$, it also holds for all $\underline{w}$. Setting $\underline{w}=0,(25)$ can be written as $a(1-z)^{a-1}(z+(1-z) \log [1-z])+z(1-$ $(1-z)^{a-1}+a z / \theta-1>0$ and a three-dimensional plot of this expression shows that the inequality is always satisfied. 
This result, illustrated in Figure 8, shows that workers send too many applications in equilibrium. The left graph shows a comparison of the equilibrium number of applications $a^{*}(\theta)$ and the efficient amount $a^{S B}(\theta)$. The right graph shows the consequences of excessive applications on the unemployment rate.

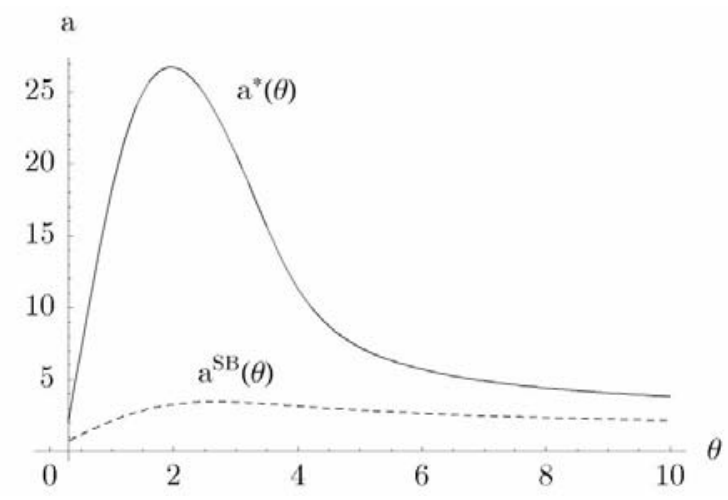

(a) Number of applications

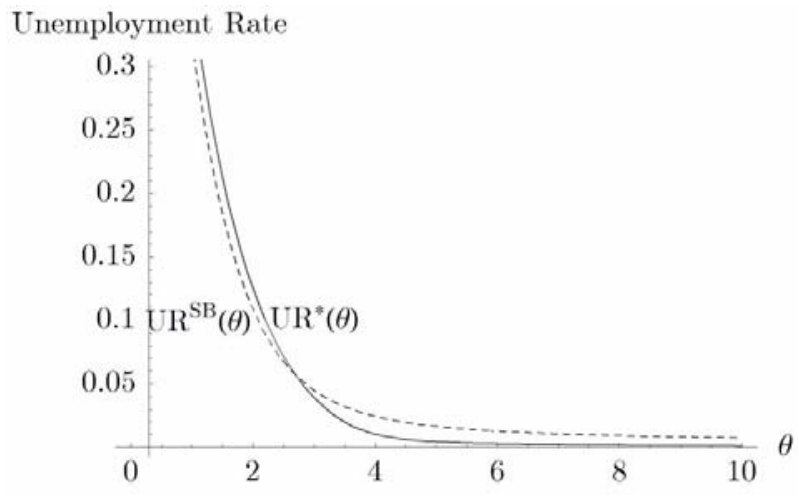

(b) Unemployment rate

Figure 8: Private and social incentives to send applications $(k=0.01, \underline{w}=0)$

To get some intuition for this result, suppose that the minimum wage is set equal to 1 . In this case wage dispersion is fully eliminated and the only reason to send applications is to increase the chance to get a job. A comparison between (20) and (24) when $\underline{w}=1$ reveals that there are too many applications in equilibrium. The reason is that an individual worker does not take into account the externalities his/her applications cause on the hiring probability of other workers (the second term of (24) is negative). When the minimum wage is set below 1 , there is wage dispersion and hence workers have an additional motive to send applications, namely, to increase their expected wage. As we mentioned before, the minimum wage can thus play a useful role in reducing congestion externalities by compressing the wage distribution and reducing the equilibrium number of applications.

This discussion suggests that the only way to get an efficient outcome in this economy is to eliminate wage dispersion. Suppose the government fixed a mandatory wage $\widehat{w}$. Using (16), workers' expected utility would be $E U\left(a_{l} ; a\right)=\left(1-(1-z(a))^{a_{l}}\right) \widehat{w}$. A comparison of the marginal gains from applications in this case with the efficiency condition (24) leads to the following result.

Proposition 8 For any given $\theta, k$, let $\widehat{a}$ be the solution to (24) and let $\widehat{z}=z(\theta, \widehat{a})$. Then there exists a mandatory wage $\widehat{w}=\frac{1}{1-\widehat{a}(1-\widehat{z})^{\widehat{a}-1}\left(\frac{1-\widehat{z}}{\widehat{a}}-\frac{\hat{z}}{\theta}\right)}$ for which the market equilibrium is (second-best) 


\section{efficient.}

\section{$6 \quad$ Free entry of vacancies}

In this Section, we examine the free-entry long-run equilibrium. We start with a consideration of firm entry for an exogenously given number of applications $a$. Let $c$ be the cost of opening a vacancy. For a given number of workers $u$, and a given number of applications $a$, the free entry equilibrium is given by the vacancy-to-unemployment ratio $\theta$ that solves $E \pi^{i}(w ; F(\cdot))-c=0$ (along with the equilibrium wage setting behavior given in Proposition 4). Or, using (11),

$$
\frac{a}{\theta} z(\theta, a)(1-z(\theta, a))^{a-1}(1-\underline{w})=c
$$

For convenience, we rewrite this free-entry condition as:

$$
\log \left[\frac{a z(\theta, a)}{\theta}\right]+(a-1) \log [1-z(\theta, a)]+\log [1-\underline{w}]=\log [c]
$$

Let us denote the LHS of this expression as $\Theta(\theta, a)$. We first note that (27) has a unique solution in $\theta$ for any given set of feasible parameters. This follows from the following three observations: (i) $\lim _{\theta \rightarrow 0} \Theta(\theta, a)=0,($ ii $) \lim _{\theta \rightarrow \infty} \Theta(\theta, a)=-\infty$ and

$$
\text { (iii) } \frac{d \Theta(\theta, a)}{d \theta}=\frac{(a-1)\left(1-e^{\frac{a}{\theta}}\right)^{2} \theta^{2}+a^{2}\left(e^{\frac{a}{\theta}}+\theta\left(1-e^{\frac{a}{\theta}}\right)\right)}{\left(1-e^{\frac{a}{\theta}}\right) \theta^{2}\left(a e^{\frac{a}{\theta}}+\theta\left(1-e^{\frac{a}{\theta}}\right)\right)}<0 \text { for all } a \geq 2 .
$$

Inspection of the free-entry condition (27) immediately shows that the long-run number of vacancies decreases in the minimum wage, for any given $a$. The intuition is simple: entry becomes less profitable as $\underline{w}$ gets larger.

We now ask how the free-entry number of vacancies depends on workers search activity. To answer this question we examine how the gains from entry change with $a$. This is given by the following derivative:

$$
\frac{d \Theta(\theta, a)}{d a}=\frac{-(a-1)\left(a+\theta\left(1-e^{\frac{a}{\theta}}\right)\right)}{a\left(a e^{\frac{a}{\theta}}+\theta\left(1-e^{\frac{a}{\theta}}\right)\right)}+\frac{-1}{\theta\left(1-e^{\frac{a}{\theta}}\right)}+\log [1-z(\theta, a)] .
$$

The sign of this expression depends in a complicated way on $a$ and $\theta$ : the first term is positive, while the second and the third terms are negative. However, it can be seen that in a neighborhood of $a=2, d \Theta(\theta, a) / d a$ is always negative. As a result, $\theta$ is initially decreasing in $a$. Numerical analysis of (28) reveals that its sign can become positive for a relatively high $a$ and intermediate levels of $\theta$. We have numerically calculated the free entry number of vacancies as a function of $a$; 
the results reveal that the number of vacancies that open in the free-entry equilibrium is typically non-monotonic in $a$. Figure 9 shows $\theta$ as a function of $a$ for various levels of the minimum wage. It can be seen that $\theta$ initially falls sharply with $a$ and then it can slightly increase in $a$. The intuition is the following. Inspection of the LHS of equation (26) reveals that an increase in $a$ leads to two effects. The first effect is positive and tells us that the likelihood to get candidates is increasing in $a$, while the second effect is negative and reflects the probability that one or more rival firms also consider your candidate, which increases the equilibrium wage and reduces profits. These effects are relatively important for low values of $a$ but become insignificant as $a$ gets large. The exponent $(a-1)$ makes the second effect vanish faster than the first effect and this explains the result.

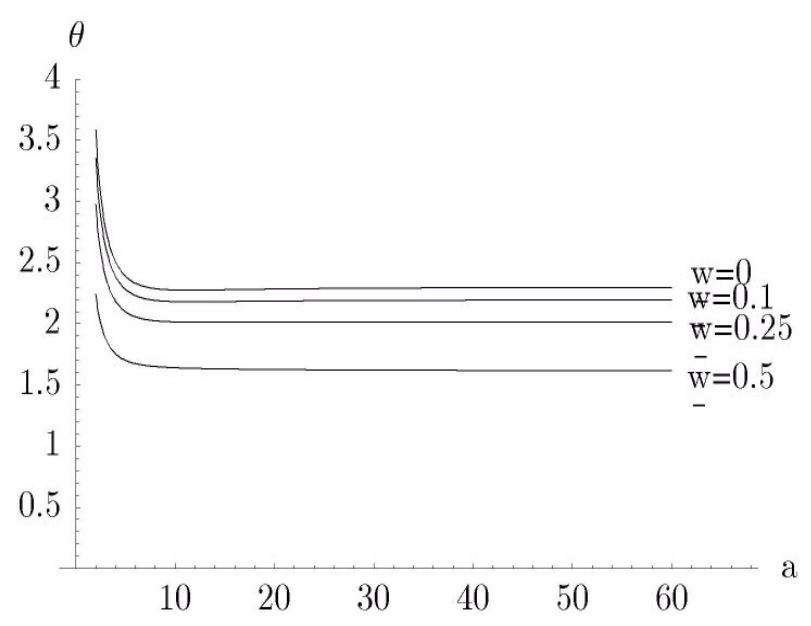

Figure 9: Free entry number of vacancies (for fixed $a, c=0.1$ ).

We now look at the free-entry equilibrium when workers are active and decide how many applications to send to maximize their expected utility. Taking into account equilibrium wage setting (Proposition 4), a free-entry equilibrium is then characterized by the solution to the system of equations (20) and (26). We note that both equilibrium conditions (20) and (26) are nonmonotonic and this makes it very difficult to characterize sets of parameters $(c, k, \underline{w})$ for which the existence and uniqueness of a long-run equilibrium is guaranteed. This is something we will not pursue in this paper. However, to gain some insight into this issue we have simulated the economy for multiple parameter values $(c, k$ and $\underline{w})$. We find that when application costs are low compared to entry costs, a free-entry equilibrium with endogenous applications always exists and is uniquely determined. (When entry costs are very low and application costs are large, there may be existence 
problems.) Figure 10 illustrates a market where both entry and application costs are relatively low $(c=k=0.01$ and $\underline{w}=0)$. The curve labelled " $U^{\prime}(a)-k$ " is the workers equilibrium condition (20), while the curve labelled " $E \pi=0$ " is the zero-profits condition. It can be seen that the curves intersect only once, at the point marked "Eq." In this example, the long run equilibrium is given by the pair $\left(a^{*}, \theta^{*}\right)=(20.5,4.3)$.

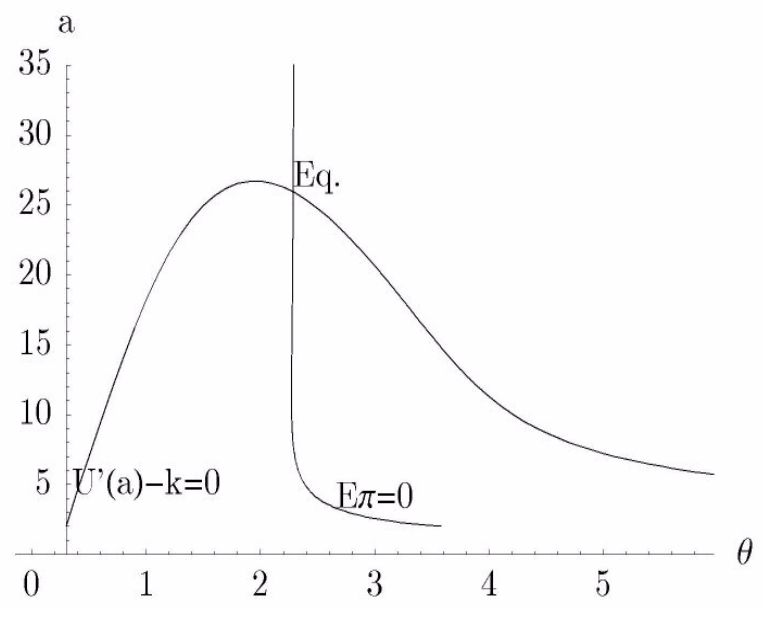

Figure 10: Free-entry equilibrium $(c=k=0.01$ and $\underline{w}=0)$

Using Figure 10, it is straightforward to ascertain the influence of parameters $c$ and $k$ on the long-run equilibrium. A decrease in entry $\operatorname{cost} c$ shifts the free entry condition to the right. In markets with very few vacancies $(\theta$ small), this results in a larger number of firms and in more applications. By contrast, in markets with many vacancies (like in Figure 10), the number of applications decreases. Similarly, we can ask how a decrease in application cost $k$ affects the longrun equilibrium. We note that this change shifts the workers' equilibrium condition upwards, which results in a larger number of applications and in entry of vacancies.

Next, we are interested in how the minimum wage $\underline{w}$ influences the long-run equilibrium. We note that this influence is highly sensitive to the initial market conditions. First, we have seen how an increase in the minimum wage can lead to more or less applications, depending on the number of vacancies $\theta$ (Proposition 6). Second, an increase in $\underline{w}$ affects not only the workers' equilibrium condition but also the free entry condition. This is illustrated in Figure 11, where $c=k=0.01$. The solid curves correspond to a situation where $\underline{w}=0$, while the dashed curves show the case $\underline{w}=0.2$. The graph reveals the following feature of the free-entry equilibrium: an increase in the minimum 
wage $\underline{w}$ always leads to exit and, if initially there are sufficiently many firms in the market, to more applications; otherwise to fewer applications. This surprising result stems from the fact that if $\theta$ is large, many wage offers are already close to the competitive wage and further increases of the minimum wage therefore reduce the marginal gains of additional applications.

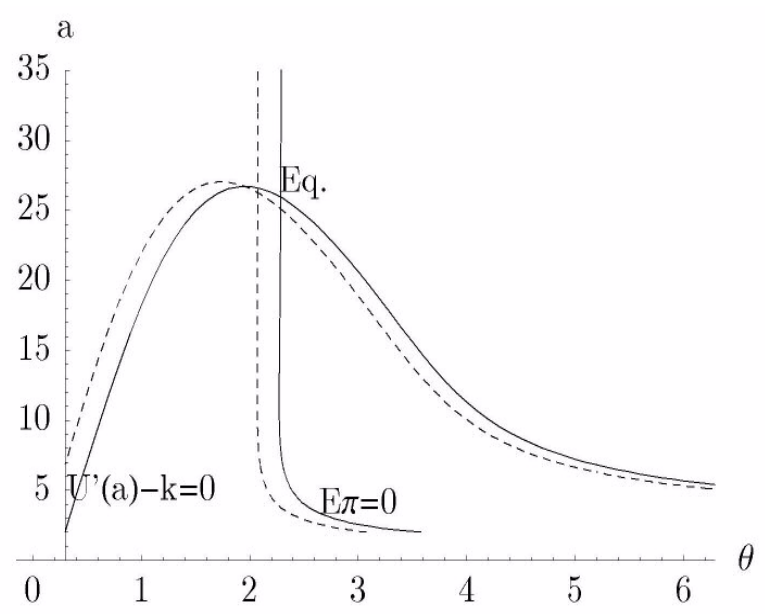

Figure 11: Free entry equilibrium for $\underline{w}=0$ (solid curves) and $\underline{w}=0.2$ (dashed curves).

The last issue we investigate is whether the free-entry long-run equilibrium is constrained efficient. We know already that the answer to this question is negative because the private incentives to send applications are excessive for any $\theta$. However, we still can ask whether the free-entry equilibrium number of firms is excessive or insufficient from a social welfare perspective. The planner's problem consists of maximizing $\Lambda-c \theta$, where $\Lambda$ is defined in equation (23). For a fixed $a$, the optimal vacancy-to-unemployment ratio is then given by the following first order condition:

$$
\frac{d \Lambda}{d \theta}=a(1-z(\theta, a))^{a-1} \frac{d z(\theta, a)}{d \theta}-c=0
$$

Or, substituting the expression for $d z / d \theta$ :

$$
\frac{d \Lambda}{d \theta}=a(1-z(\theta, a))^{a-1}\left[\frac{z(\theta, a)}{\theta}-\frac{\exp \left(-\frac{a}{\theta}\right)}{\theta}\right]-c=0
$$

Comparing (26) with (30) reveals that firms incentives to enter are not socially efficient. An efficient outcome requires

$$
z(\theta, a)(1-\underline{w})=z(\theta, a)-\exp \left(-\frac{a}{\theta}\right)
$$

which implies that: 
Proposition 9 For any given number of applications a, there exists a sufficiently low minimum wage $\underline{\widehat{w}}$ such that for all $\underline{w} \leq \underline{\widehat{w}}$ firms incentives to enter the labor market are excessive; moreover, there exists a sufficiently large minimum wage $\underline{\widetilde{w}}$ such that for all $\underline{w} \geq \underline{\widetilde{w}}$ firms incentives to enter the labor market are insufficient.

\section{Final remarks}

We presented a simple strategic wage setting game where workers must decide upon the number of jobs they apply for and firms simultaneously post a wage taking into account the effect that the wage has on the behavior of workers and where workers similarly take into account the effect that their search intensity has on wages. Quite surprisingly, this model generates, even in the most simple setting, many interesting insights. We show that an equilibrium where workers apply to just one job exhibits unemployment and absence of wage dispersion; this type of Diamond result cannot be sustained generally. An equilibrium where workers apply for two or more jobs typically exhibits wage dispersion and unemployment. In addition, we showed that there exists a first order stochastic dominance relation between wage distributions of labor markets with different vacancy-to-unemployment ratios and minimum wages; however average wage is non-monotonic in the number of applications. Finally, we showed that the resulting equilibrium is not efficient. Workers cause too much congestion by sending out too many applications. Introduction of a minimum wage can improve welfare when the vacancy-to-unemployment ratio is large since it reduces the number of applications but the only way to align the private and the social incentives is to impose a mandatory wage that eliminates wage dispersion altogether.

\section{References}

[1] Acemoglu, D. And R. Shimer (2000), Wage and Technology Dispersion, Review of Economic Studies 67, 585-608.

[2] Albrecht, J.W. And B. Axell (1984), An equilibrium model of search unemployment, Journal of Political Economy, 92, 824-40.

[3] Albrecht, J.W., P.A. Gautier, and S.B. Vroman (2002), Equilibrium Directed Search with Multiple Applications, mimeo Tinbergen Institute.

[4] Albrecht, J.W, P.A Gautier, and S.B. Vroman (2003), Matching with Multiple Applications, the Limiting Case, mimeo, Tinbergen Institute. 
[5] Berg van den, G.J. (2003), Multiple equilibria and minimum wages in labor markets with informational frictions and heterogeneous production technologies, International Economic Review, 44-4, 1337.

[6] Brown, C. (1999), Minimum wages, employment, and the distribution of income, chapter 32 in Handbook of labor Economics, O. Ashenfelter and D. Card ed,2101-2163, North Holland.

[7] Burdett, K. And K. L. Judd (1983), Equilibrium Price Dispersion, Econometrica 51, 4, 955-969.

[8] Burdett, K. And D. Mortensen (1998), Wage Differentials, Employer Size and Unemployment, International Economic Review, 39, 257-73.

[9] Butters (1977), Equilibrium Distributions of Sales and Advertising Prices, Review of Economic Studies, 44-3, 465-491.

[10] Burdett, K., S. Shi, And R. Wright (2001), Pricing and Matching with Frictions, Journal of Political Economy, 109, 1060-85.

[11] Dasgupta, P. And E. Maskin (1986), "The Existence of Equilibrium in Discontinuous Economic Games, Part I (Theory)," Review of Economic Studies 53(1), 1986, pp.1-26.

[12] Diamond, P. (1971), A Model of Price Adjustment, Journal of Economic Theory, 3, 156-68.

[13] Janssen M. and J.L. Moraga-Gonzalez (2003), Strategic Pricing, Consumer Search and the Number of Firms, Review of Economic Studies, forthcoming.

[14] Kuhn, P. And M. Skuterud (2004), Internet Job Search and Unemployment Durations, American Economic Review, 94-1, 218-232.

[15] LANG, K. (1991), Persistent wage dispersion and involuntary unemployment, The Quarterly Journal of Economics, 106, 181-202.

[16] LeE, D.S. (1999), Wage inequality in the United States during the 1980's: Rising dispersion or falling minimum wage?, Quarterly Journal of Economics, 114, 941-1023.

[17] Montgomery, J. (1991), Equilibrium wage dispersion and interindustry wage differentials, Quarterly Journal of Economics, 106, 163-179.

[18] Pissarides, C. (2000), Equilibrium Unemployment Theory, $2^{\text {nd }}$ ed., MIT Press.

[19] Rothschild, M. (1973), Models of market organization with imperfect information: a survey, Journal of Political Economy, LXXXI, 1283-1308.

[20] Shimer, R. (2004), Search intensity, mimeo, University of Chicago.

[21] Teulings, C.N. (2003), Contributions of minimum wages to increasing wage inequality, Economic Journal, 113, 801-33. 


\section{Appendix}

\section{A Proof of Proposition 1}

(i) Since workers only observe the wage after they have applied to a firm, firms only have an incentive to offer a worker $w>\underline{w}$ when the probability that the worker also gets an offer from a competing firm is strictly positive. When $a=1$ this occurs with probability 0 , hence no firm has an incentive to offer $w>\underline{w}$. So when $a=1$ the equilibrium wage distribution must be degenerate at $w=\underline{w}$.

(ii) Consider now the case in which $\min \{u, v\}>a \geq 2$ in equilibrium. We prove the statement above by contradiction. Assume that all firms post some wage $w$ in $[\underline{w}, 1)$ in equilibrium. The payoff of a firm depends positively on the probability $\rho$ that a vacancy succeeds in hiring a worker and negatively on the posted wage. First, we show that a vacancy remains vacant with a strictly positive probability, i.e., $\rho<1$. When $u<v$, this is easy because then $\rho \leq \frac{u}{v}<1$, for all $a$. If $u \geq v$, a firm with applicants fails to hire a worker when all its applicants get hired by other firms. To show that $\rho<1$ also in this case, it is enough to find an event that can occur with positive probability where some firm $i$ with applicants fails to fill its vacancy. An example of such an event is the case where some firm $i$ only gets a single application from one of the workers, say $j$. It is obvious that this event can indeed occur with positive probability, for all $2 \leq a \leq v-1$ and for any $u$. Suppose this event is realized; then there is a positive probability that this applicant $j$ gets hired by some other firm different than $i$; as a result $\rho<1$. Second, we establish a contradiction. Note that equilibrium the profits to firm $i$ are $\pi_{i}=\rho(1-w)$. We now show that firm $i$ can do better by deviating and offering a higher wage, say $w+\varepsilon$. If this is so, the firm in question will always succeed in hiring a worker, provided that it obtains at least an application. This occurs with probability $1-\left(1-\frac{a}{v}\right)^{u}$, which is greater than $\rho .{ }^{13}$ By deviating, the firm would make profits $\pi_{i}^{D}=\left[1-\left(1-\frac{a}{v}\right)^{u}\right](1-w-\varepsilon)$, which are strictly higher than $\pi_{i}$ for sufficiently small $\varepsilon>0$. This argument rules out all wages in $[\underline{w}, 1)$. It remains to be proved that a wage distribution degenerated at $w=1$ cannot be an equilibrium either when $\min \{u, v\}>a \geq 2$. Note that in this case firms would make zero profits in equilibrium. If a firm deviates and offers a lower wage, say $\underline{w}$, this firm would obtain a profit $\pi_{i}^{D}=\rho^{D}(1-\underline{w})$, where $\rho^{D}$ is the probability that the deviant is matched with a worker. To rule out this case we need to show that $\rho^{D}>0$. Consider first that $u<v$. To prove that $\rho^{D}>0$, it is sufficient to give an example of an event that can arise with positive probability where the deviant hires a worker, for $2 \leq a \leq u$. This is easy because even if the deviant gets only one applicant, there is a positive probability that this candidate gets no offers from any of the other firms to which she applied for a job. Therefore, $\rho^{D}>0$ when $u<v$ and a firm would gain by deviating. Next consider $u \geq v$. Let us number the vacancies from 1 to $V-1$ and the workers from 1 to $U$. Once again, to prove that $\rho^{D}>0$, it is sufficient to find a positive-probability event in which the deviant hires a worker for sure, now for $2 \leq a \leq v-1$. An example of such an event is the case where worker $j$ applies for vacancy $i=j$, for the deviant's vacancy and possibly for some other vacancies and worker 1 is picked by vacancy 1 , worker 2 is picked by vacancy $2, \ldots$, and worker $V-1$ is picked by vacancy $V-1$. In that case, the deviant hires worker $V$ (or any other worker from $V$ to $U$ ) with probability 1 . Therefore, the deviant would gain by deviating. So we have established that if $\min \{u, v\}>a \geq 2$, there is no wage equilibrium in pure strategies. The same type of arguments can be used to show that this also holds when $a=u<v$. We finally argue that if an equilibrium wage distribution $F(w)$ exists, it must be atomless. The proof follows the arguments above and we only sketch it here. Suppose, on the contrary, that there were atoms in the

\footnotetext{
${ }^{13}$ Since $p=\left[1-\left(1-\frac{a}{v}\right)^{u}\right][1-\operatorname{Pr}($ all candidates get hired by other firms $)]$.
} 
wage distribution and consider a firm offering a wage $\widehat{w}$ such that $d F(\widehat{w})>0$. Then, with strictly positive probability, such firm would tie with all other firms at offering $\widehat{w}$, and as shown before, the firm would gain by offering a slightly higher wage. An atom at the competitive wage cannot arise in equilibrium either because a firm offering such wage would have an incentive to deviate and offer a lower one. Those arguments hold for any recruiting technology $R_{m}$. In addition, under $R_{1}, \ldots, R_{u-1}$ they also hold for $v>a>u$.

(iii) Under $R_{u}$. If $a=v \leq u$, each firm gets $u$ applications and hires a worker with probability 1 and no firm has an incentive to offer a wage above the minimum wage. Under $R_{1}, \ldots, R_{u-1}$, there is always competition for workers and therefore the arguments in (ii) also hold here. This completes the proof.

\section{Proof of Proposition 3}

In this appendix we calculate a sufficient condition for an equilibrium with $a=1$ to exist. The first condition is that participation yields non-negative payoffs. Hence, $\gamma_{1}(\cdot) \underline{w} \geq k$. The second condition is that deviating to $a=2$ is not profitable. To calculate the payoffs from such a deviation we must first calculate the probability to get a job offer when applying for 2 jobs when the other workers apply to one job. Let $E_{1}$ be the probability that the deviant's first application results in an offer and $E_{2}$ be the probability that his second application results in an offer. Note that $P\left(E_{1}\right)=\hat{z}=\frac{\widehat{\rho} v}{u+1}=\frac{\left(1-\left(1-\frac{1}{v}\right)^{u-1}\left(1-\frac{2}{v}\right)\right) v}{u+1}$, where $\widehat{\rho}=\left(1-\left(1-\frac{1}{v}\right)^{u-1}\left(1-\frac{2}{v}\right)\right)$ is the probability that a vacancy has applications (one minus the probability that non-deviant workers do not apply minus the probability that the deviant does not apply). The probability that at least one of the deviant's applications results in an offer is $1-P\left(\bar{E}_{1}\right) P\left(\bar{E}_{2} \mid \bar{E}_{1}\right)$ which is only in the limit $\left(u, v \rightarrow \infty, \frac{v}{u} \rightarrow \theta\right)$ equal to $1-P\left(\bar{E}_{1}\right)^{2}$, see Albrecht et al. (2004). A negative dependency is a general property of finite urn-ball type of models: if a subset of the urns receives many balls then a disjoint subset of the urns must receive a few balls. Hence the fact that the first application does not result in a match gives implicit information on the total number of applications the firm where this application landed had. The likelihood that the second one does not result in an offer therefore becomes smaller. If $E_{1}, \ldots, E_{n}$ are negatively associated: ${ }^{14}$

$$
P\left(\bar{E}_{1}, \ldots, \bar{E}_{n}\right) \leq\left(P \bar{E}_{1}\right)^{n},
$$

Next, we turn to the proof. Assume for the moment that $a=1$ in equilibrium. First note that an applicant gets either a single wage offer or no wage offer at all. As a result, a firm offering a wage to a worker faces no competition from other firms to hire such worker. This implies that firms have no incentives to offer wages above the minimum wage since doing so does not increase the hiring probability. Hence, the equilibrium payoff to a firm is $\pi_{i}=\rho(1-\underline{w})$, where $\rho$ is the probability of succeeding in hiring a worker. Note that the number of applications that a firm receives is a random variable with a $\operatorname{bin}(u, a / v)$ distribution. Then, $\rho$ is the standard "urn-ball" probability to get one or more applicants, i.e., $\rho=1-\left(1-\frac{1}{v}\right)^{u}$.

We now check that $a=1$ is indeed a worker's best-response. In equilibrium, the expected utility for a worker is equal to $U^{e}=z \underline{w}-k$, where $z$ is the probability that a worker gets a job. This probability can be easily computed, $z=\frac{\rho v}{u}$. Expected utility in equilibrium is non-negative if and only if the condition $k \leq \gamma(\underline{w} ; u, v)$ holds. A potential deviant worker who sends out 2 applications

\footnotetext{
${ }^{14}$ I.e. for the $3 \times 3$ case, $\left(1-P\left(\bar{E}_{1}\right)^{2}\right)=\frac{1127}{1296}$ while $1-P\left(\bar{E}_{1}\right) P\left(\bar{E}_{2} \mid \bar{E}_{1}\right)=\frac{11}{12}$.
} 
will get a job with a probability that is at least $1-(1-\hat{z})^{2}$ (where $\hat{z}$ is given above). So her expected utility is at least $U_{d}^{e}(a=2)=\left(1-(1-\hat{z})^{2}\right) \underline{w}-2 k$. For a symmetric equilibrium, we need that workers have no incentives to deviate, i.e. it must be the case that $z \underline{w}-k \geq\left[1-(1-\hat{z})^{2}\right] \underline{w}-2 k$, or $k \geq\left[1-(1-\hat{z})^{2}\right] \underline{w}-\gamma_{1}(\underline{w} ; u, v)$. Finally, the upper bound comes from setting $\hat{z}=1$. We note that this condition also ensures that a worker does not find it beneficial to apply to 3 or more vacancies. The proof is now complete.

\section{B Hiring probabilities for $R_{3}(v=3, u=3, a=2)$}

\section{B.1 Derivation of $p^{n: 3}$}

$p^{1: 3}, p^{2: 3}$ and $p^{3: 3}$ are the probabilities that a firm offering the highest, second highest and lowest wage in the market hires a worker, respectively. Note that $p^{1: 3}=1-\left(1-\frac{2}{3}\right)^{3}=\frac{26}{27}$. To calculate $p^{2: 3}$, note that the second highest wage firm always hires a worker when it has 2 or more applicants and it remains vacant when it has one applicant and this applicant gets hired by the highest wage firm. The likelihood that the candidate at the second highest wage firm also applied to the highest wage firm is $\frac{1}{2}$. Given that the candidate in question is the only applicant at the second highest wage firm and he also applied to the highest wage firm, there must be 3 candidates at the highest wage firm so he gets hired by that firm with probability $\frac{1}{3}$. The probability that a firm gets exactly 1 candidate is $\left(\begin{array}{l}3 \\ 1\end{array}\right)\left(\frac{2}{3}\right)\left(\frac{1}{3}\right)^{2}=\frac{6}{27}=\frac{2}{9}$, and the probability to get 2 or more candidates is: $1-\frac{1}{27}-\frac{6}{27}=\frac{20}{27}$. Thus

$$
p^{2: 3}=\frac{1}{27} 0+\frac{6}{27}\left(\frac{1}{2}\left(1-\frac{1}{3}\right)+\frac{1}{2} 1\right)+\frac{20}{27} 1=\frac{25}{27}
$$

To calculate $p^{3: 3}$, note that a firm always hires a worker when it gets 3 applicants, which happens with probability $\frac{8}{27}$; a firm fails to hire a worker when all its candidates get hired by the highest and/or the second highest wage firm. Thus:

$$
p^{3: 3}=\frac{1}{27} 0+\frac{6}{27}\left(\frac{1}{2}\left(\frac{1}{2} 1+\frac{1}{2} 0\right)+\frac{1}{2}\left(\frac{2}{3} \frac{1}{2}+\frac{1}{3} 0\right)\right)+\frac{12}{27}\left(\frac{1+1+\frac{3}{4}+\frac{3}{4}}{4}\right)+\frac{8}{27} 1=\frac{21}{27}
$$

\section{B.2 Derivation of $q^{n: 3}$}

The probabilities of being hired at respectively the 1st, 2nd or 3rd highest wage, $q^{1: 3}, q^{2: 3}$ and $q^{3: 3}$, can be calculated as follows. First note that a worker applies to vacancy $A$ with probability $\frac{2}{3}$ and, conditional on applying, she is the only applicant at vacancy $A$ with probability $\frac{1}{9}$ (workers 2 and 3 both have a $\left(\frac{1}{3}\right)^{2}$ chance to not apply to the same vacancy worker 1 has applied to). With probability $\frac{4}{9}$ she is one of the two candidates at $A$, and with probability $\frac{4}{9}$ she is one of the 3 candidates. Hence:

$$
\begin{aligned}
& q^{1: 3}=\frac{2}{27}\left(1+4 \cdot \frac{1}{2}+4 \cdot \frac{1}{3}\right)=\frac{26}{81} \\
& q^{2: 3}=\frac{1}{27}\left(\frac{1}{3}+2 \cdot \frac{1}{2}+4 \cdot \frac{1}{4}+\frac{2}{3}+0\right)+\frac{1}{27}\left(5 \cdot \frac{1}{2}+2 \cdot \frac{3}{4}+\frac{1}{3}+1\right)=\frac{25}{81} \\
& q^{3: 3}=\frac{1}{27}\left(5 \cdot \frac{1}{2}+2 \cdot \frac{1}{4}+\frac{1}{3}+0\right)+\frac{1}{27}\left(\frac{2}{3}+6 \cdot \frac{1}{2}+\frac{1}{3}+0\right)=\frac{22}{81}
\end{aligned}
$$


The worker remains unemployed with probability $1-q^{1: 3}-q^{2: 3}-q^{3: 3}=16 / 162$.

\section{B.3 Derivation of $q_{3}^{n: 3}$ and $q_{1}^{n: 3}$}

Let $q_{3}^{1: 3}$ be the probability that a deviant worker who sends 3 applications gets the highest wage; likewise, let $q_{3}^{2: 3}$ and $q_{3}^{3: 3}$ be the probability that she gets the second highest wage and the lowest wage, respectively. Standard combinatorics yields:

$$
\begin{aligned}
q_{3}^{1: 3} & =\frac{1}{9} 1+\frac{4}{9} \frac{1}{2}+\frac{4}{9} \frac{1}{3}=\frac{13}{27} \\
q_{3}^{2: 3} & =\frac{1}{9} \frac{2}{3}+\frac{4}{9}\left(\frac{\frac{1}{2} 2+\frac{1}{4} 2}{4}\right)+\frac{4}{9}\left(\frac{0+\frac{1}{4} 2+\frac{1}{3}}{4}\right)=\frac{1}{3}, \\
q_{3}^{3: 3} & =\frac{1}{9} \frac{1}{3}+\frac{4}{9}\left(\frac{\frac{1}{4} 2+\frac{1}{6} 2}{4}\right)+\frac{4}{9}\left(\frac{0 \cdot 2+\frac{1}{4} 2}{4}\right)=\frac{5}{27} .
\end{aligned}
$$

In this case, the deviant gets a job for sure. Consider a worker who deviates and sends out just one application. As before, let $q_{1}^{1: 3}$ be the probability that a deviant worker gets the maximum wage, $q_{1}^{2: 3}$ be the probability she gets the second highest wage, and $q_{1}^{3: 3}$ be the probability to get the lowest wage.

$$
\begin{aligned}
& q_{1}^{1: 3}=\frac{1}{3}\left(\frac{1}{9} 1+\frac{4}{9} \frac{1}{2}+\frac{4}{9} \frac{1}{3}\right)=\frac{13}{81} \\
& q_{1}^{2: 3}=\frac{1}{3}\left(\frac{1}{9} 1+\frac{4}{9}\left(\frac{\frac{1}{2} 2+\frac{3}{4} 2}{4}\right)+\frac{4}{9}\left(\frac{\frac{1}{2} 3+\frac{1}{3}}{4}\right)\right)=\frac{16}{81} \\
& q_{1}^{3: 3}=\frac{1}{3}\left(\frac{1}{9} 1+\frac{4}{9}\left(\frac{\frac{3}{4} 2+1 \cdot 2}{4}\right)+\frac{4}{9}\left(\frac{1 \cdot 2+\frac{1}{2} 2}{4}\right)\right)=\frac{5}{18} .
\end{aligned}
$$

In this case the deviant worker remains unemployed with probability 59/162.

\section{Derivation of equilibria under $R_{1}$ for $(u=3, v=3)$}

Proposition 3 shows that when workers apply for just one job, firms offer the minimum wage for any recruiting technology. As a result, the equilibrium characterization for $a=1$ is identical to that in the $R_{3}$ case.

Next, take $a=2$. This generates a wage-dispersed equilibrium. To derive the payoff to a firm $i$ offering a wage $w$ when the rivals offer a random draw from $F(w)$, we consider the following events. First, note that the number of applicants a firm receives is $\operatorname{bin}\left(u, \frac{a}{v}\right)$, so the probability firm $i$ has no applicants is $1 / 27$, that it has 1 applicant is $6 / 27$, that it has two applicants is $12 / 27$ and that it has 3 applicants is $8 / 27$. If firm $i$ has one applicant, the other firm where this candidate applied to has in total 3 applications with probability 1 . If firm $i$ has 2 candidates, the firm picks one of them at random and makes an offer to him/her. It may be the case that the chosen worker is queueing together with the two other workers in the other firm he applied for, which happens with probability $1 / 2$ and in this case firm $i$ hires the worker with probability $2 / 3+1 / 3 F(w)$; or it may be the case that the chosen worker has just one competitor for the offer of the other firm he applied for, which happens with probability $1 / 2$ and in this case firm $i$ hires the worker with probability $1 / 2+1 / 2 F(w)$. If firm $i$ has three applicants is, the firm picks one of them at random 
again and makes an offer to him/her. The chosen applicant may be queueing with no other worker in the other vacancy he applied for, which happens with probability $1 / 4$ and in this case firm $i$ hires the worker with probability $F(w)$; or it may be the case that the chosen worker is queueing with just one other worker in the other vacancy he applied for, which happens with probability $2 / 4$ and in this case firm $i$ hires the worker with probability $1 / 2+1 / 2 F(w)$; or, finally, it may be the case that the chosen worker is queueing with two other workers in the other vacancy he applied for, which happens with probability $1 / 4$ and in this case firm $i$ hires the worker with probability $2 / 3+1 / 3 F(w)$.

Collecting terms gives the following payoff:

$$
\begin{aligned}
\pi_{i}(w ; F(w) & =\left[\frac{6}{27}\left(\frac{2}{3}+\frac{1}{3} F(w)\right)+\frac{12}{27}\left(\frac{1}{2}\left(\frac{2}{3}+\frac{1}{3} F(w)\right)+\frac{1}{2}\left(\frac{1}{2}+\frac{1}{2} F(w)\right)\right)+\right. \\
& \left.\frac{8}{27}\left(\frac{1}{4}\left(\frac{2}{3}+\frac{1}{3} F(w)\right)+\frac{2}{4}\left(\frac{1}{2}+\frac{1}{2} F(w)\right)+\frac{1}{4} F(w)\right)\right](1-w) \\
& =\frac{6}{27} \frac{2}{3}+\frac{12}{27}\left(\frac{1}{2} \frac{2}{3}+\frac{1}{2} \frac{1}{2}\right)+\frac{8}{27}\left(\frac{1}{4} \frac{2}{3}+\frac{2}{4} \frac{1}{2}\right) \\
& +\left(\frac{6}{27} \frac{1}{3}+\frac{12}{27}\left(\frac{1}{2} \frac{1}{3}+\frac{1}{2} \frac{1}{2}\right)+\frac{8}{27}\left(\frac{1}{4} \frac{1}{3}+\frac{2}{4} \frac{1}{2}+\frac{1}{4}\right)\right) F(w) \\
& =\left(\frac{43}{81}+\frac{35}{81} F(w)\right)(1-w)
\end{aligned}
$$

Note that when firm $i$ offers the highest possible wage in the market it fails to hire a worker with probability $3 / 81$. Setting $w=\underline{w}$ yields equilibrium profits: $\pi_{i}(\underline{w})=\frac{43}{81}(1-\underline{w})$. Let $\bar{w}$ denote the upper bound of the wage distribution. In a mixed strategy equilibrium, the firm must be indifferent between offering any wage in $[\underline{w}, \bar{w}]$ so $F(w)$ must solve:

$$
\left(\frac{43}{81}+\frac{35}{81} F(w)\right)(1-w)=\frac{43}{81}(1-\underline{w})
$$

which yields (4). Setting $F(\bar{w})=1$ and solving for $\bar{w}$ one finds the upper bound of the wage distribution.

Let $E_{1}, E_{2}, E_{3}$ denote the expected maximum wage from a sample of size 1,2 , and 3 , respectively. ${ }^{15}$ The expected payoff to a worker in equilibrium is given by:

$$
U^{e}(a=2)=\frac{86}{162} E_{1}+\frac{35}{162} E_{2}-2 k
$$

where $86 / 162$ is the probability that the worker receives just one job offer and $35 / 162$ is the probability that he/she gets two job offers. We note that a worker gets no job with a probability equal to $41 / 162$.

In equilibrium, a worker must obtain non-negative expected utility, which yields the condition:

$$
k \leq \phi_{20}=\frac{86}{324} E_{1}+\frac{35}{324} E_{2}
$$

and there should be no profitable upward or downward deviation. The expected utility for a deviant

\footnotetext{
${ }^{15}$ For a given wage distribution $F(w)$, the expected maximum wage from a random sample of size $n$ is $E_{n}=$ $\int_{-\infty}^{\infty} n F(w)^{n-1} f(w) d w$.
} 
who sends 3 applications is given by

$$
U_{d}^{e}\left(a^{d}=3\right)=\frac{69}{162} E_{1}+\frac{60}{162} E_{2}+\frac{15}{162} E_{3}-3 k
$$

where $69 / 162$ is the probability that the worker receives just one job offer, $60 / 162$ is the probability that he/she gets two wage offers and 15/162 is the probability he/she receives three offers. We note that in this case the probability the worker remains unemployed is $18 / 162$. Deviating to $a=3$ is not profitable if

$$
\begin{aligned}
U^{e}(a & =2) \geq U_{d}^{e}\left(a^{d}=3\right), \text { or: } \\
k & \geq \phi_{23}=-\frac{16}{162} E_{1}+\frac{25}{162} E_{2}+\frac{15}{162} E_{3}
\end{aligned}
$$

Next, we have to establish when it is not profitable to deviate to $a=1$. Consider a worker who deviates and sends out just one application. The expected utility is

$$
\begin{gathered}
P(0 \text { offers })=\frac{1}{9}\left(\frac{2}{3}+\frac{2}{3}+\frac{1}{2}+\frac{2}{3}+\frac{2}{3}+\frac{1}{2}+\frac{1}{2}+\frac{1}{2}+0\right)=\frac{14}{27} \\
U_{d}^{e}(a=1)=\frac{13}{27} E_{1}-k
\end{gathered}
$$

where $13 / 27$ is the probability of getting employed. Deviating to $a=1$ is not profitable if:

$$
\begin{aligned}
U^{e}(a & =2) \geq U_{d}^{e}\left(a^{d}=1\right) \text { or: } \\
k & \leq \phi_{21}=\frac{7}{162} E_{1}+\frac{35}{162} E_{2}
\end{aligned}
$$

We note that $\phi_{20}<\phi_{21}$ so the condition (33) is implied by condition (31). So it must be the case that $\phi_{20} \geq k \geq \phi_{23}$ for an equilibrium with $a=2$ to exist.

We finally consider the case where each worker sends 3 applications. The payoff to a firm offering a wage $w$ when the rivals offer a random draw from $F(w)$ is calculated as follows. First, note that firm $i$ picks a worker at random and makes an offer to him/her. The firm hires this worker surely when the other two firms offer their vacancy to different workers, i.e., with probability $(2 / 3)^{2}$. Second, with probability $2(1 / 3)(2 / 3)$ at least one other firm offers its job to the worker of firm $i$, in which case firm $i$ only hires if its wage is higher, i.e., with probability $F(w)$. Finally, with probability $(1 / 3)^{2}$ both other firms offer their job to the candidate of firm $i$, in which case firm $i$ only hires with probability $F(w)^{2}$. This leads to the following payoff:

$$
\pi_{i}(w ; F(w))=\left[\frac{1}{9} F^{2}(w)+\frac{4}{9} F(w)+\frac{4}{9}\right](1-w)
$$

Solving the equation $\pi_{i}(w ; F(w))=\pi_{i}(\underline{w})$ yields the distribution function provided in the text. Setting $F(\bar{w})=1$ and solving for $\bar{w}$ yields $\bar{w}=(5+4 \underline{w}) / 9$.

To calculate the expected payoff for a worker when $a=3$, note that we can think of this process as each firm applying to 1 worker so the number of offers a worker gets is $\operatorname{bin}\left(v, \frac{1}{u}\right)$. The worker receives three offers with probability $(1 / 3)^{3}$, two offers with probability $3(1 / 3)^{2}(2 / 3)$, one offer with probability $3(1 / 3)(2 / 3)^{2}$.and no offer with probability $(2 / 3)^{3}$. As a result,

$$
U^{e}(a=3)=\frac{4}{9} E_{1}+\frac{2}{9} E_{2}+\frac{1}{27} E_{3}-3 k
$$


In equilibrium, a worker must obtain non-negative expected utility, which yields the condition

$$
k \leq \Omega_{30}=\frac{4}{27} E_{1}+\frac{2}{27} E_{2}+\frac{1}{81} E_{3} .
$$

In addition, there should be no profitable downward deviation. The expected utility for a deviant who sends just 2 applications is calculated taking into account that the worker gets one offer with probability $2(1 / 3)(2 / 3)$ and two offers with chance $(1 / 3)^{2}$. We note that the worker receives no offer with probability $(2 / 3)^{2}$. This yields a payoff

$$
U_{d}^{e}\left(a^{d}=2\right)=\frac{4}{9} E_{1}+\frac{1}{9} E_{2}-2 k
$$

Deviating to $a=2$ is not profitable if:

$$
\begin{aligned}
U^{e}(a & =3) \geq U_{d}^{e}\left(a^{d}=2\right) \text { or: } \\
k & \leq \Omega_{32}=\frac{1}{9} E_{2}+\frac{1}{27} E_{3} .
\end{aligned}
$$

Deviation to $a=1$ is not profitable provided that

$$
\begin{gathered}
U^{e}(a=3) \geq U_{d}^{e}\left(a^{d}=1\right)=\frac{1}{3} E_{1}-k \text { or: } \\
k \leq \Omega_{31}=\frac{1}{2}\left[\frac{1}{9} E_{1}+\frac{2}{9} E_{2}+\frac{1}{27} E_{3}\right] .
\end{gathered}
$$

We note that $\Omega_{32}<\Omega_{31}<\Omega_{30}$ so the condition (35) implies conditions (34) and (36).

\section{Proof Corollary 1}

To prove that the expected wage is initially increasing in $a$ for all parameters, we evaluate (13) at $a=2$. This yields

$$
\begin{aligned}
\left.\frac{d E[w]}{d a}\right|_{a=2} & =\left.\frac{\partial E[w]}{\partial z} \frac{d z}{d a}\right|_{a=2}+\left.\frac{\partial E[w]}{\partial a}\right|_{a=2} \\
& =(1-\underline{w}) \int_{0}^{1} \frac{y\left(\frac{1-z(\theta, 2)}{2}-\frac{z(\theta, 2)}{\theta}\right)-(1-z(\theta, 2))(1-z(\theta, 2)(1-y)) \log \left[\frac{1-z(\theta, 2)}{1-z(\theta, 2)(1-y)}\right]}{(1-z(\theta, 2)(1-y))^{2}} d y .
\end{aligned}
$$

Note that the denominator of the integrand of this expression is positive, for all $y$. Now we show that the numerator is also positive, for all $y$. That is, we need to show that

$$
-(1-z)(1-z+z y) \log \left[\frac{1-z}{1-z+z y}\right] \geq y\left(\frac{z}{\theta}-\frac{1-z}{2}\right)
$$

for all $y$, where we have suppressed the arguments of $z(\cdot)$ to save on notation. Notice that (37) holds for $y=0$. Moreover, the LHS of (37) increases in $y$ at a rate $z(1-z)\left(1-\log \left[\frac{1-z}{1-z+z y}\right]\right)$, while the RHS does so at a constant rate $\left(\frac{1-z}{2}-\frac{z}{\theta}\right)$. Now we note that $z(1-z)\left(1-\log \left[\frac{1-z}{1-z+z y}\right]\right)>$ $z(1-z)(1-\log [1-z])>\left(\frac{z}{\theta}-\frac{1-z}{2}\right)$, for all $\theta$, where the last inequality can be easily plotted in $\theta$ using the expression for $z(\theta, 2)$ given above. Thus, the expected wage increases in the number of 
applications in a neighborhood of $a=2$, for all $\theta$.

We now prove that if $\theta$ is sufficiently large, the expected wage is increasing in the number of applications $a$. We can rewrite (13) in the following way:

$\frac{d E[w]}{d a}=(1-\underline{w}) \int_{0}^{1} \frac{(1-z)^{a-1}}{(1-z(1-y))^{a}}\left[(a-1) y\left(\frac{1}{a}-\frac{1}{\frac{a}{1-e^{-\frac{a}{\theta}}}-\theta}\right)-(1-z(1-y)) \log \left[\frac{1-z}{1-z(1-y)}\right]\right] d y$.

Notice that the sign of this derivative depends on the sign of the term between square brackets. The LHS of that term is negative, decreases as $\theta$ increases and converges to $-\frac{1}{a}$ as $\theta \rightarrow \infty$ while its RHS is positive, increases in $\theta$ and converges to infinity as $\theta \rightarrow \infty$. As a result there exists $\widetilde{\theta}$ such that for all $\theta>\widetilde{\theta}$, the sign of this derivative is positive. The proof is now complete. 